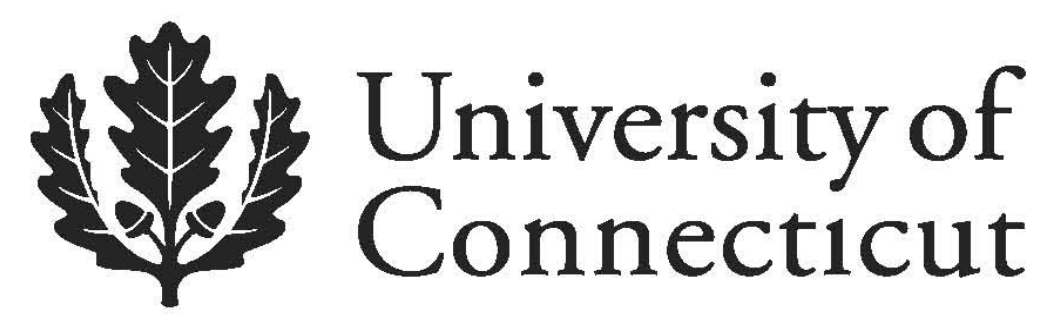

Department of Economics Working Paper Series

\title{
The Optimality and Controllability of Discretionary Monetary Policy
}

Huiping Yuan

Xiamen University

Stephen M. Miller

University of Nevada, Las Vegas and University of Connecticut

Working Paper 2011-17

August 2011

341 Mansfield Road, Unit 1063

Storrs, CT 06269-1063

Phone: (860) 486-3022

Fax: (860) 486-4463

http://www.econ.uconn.edu/

This working paper is indexed on RePEc, http://repec.org/ 


\title{
The Optimality and Controllability of Discretionary Monetary Policy
}

\author{
Huiping Yuan* \\ Xiamen University, Xiamen, Fujian 361005, China \\ E-mail: hpyuan@xmu.edu.cn \\ and \\ Stephen M. Miller** \\ University of Nevada, Las Vegas, Las Vegas, NV 89154-6005, USA \\ E-mail: stephen.miller@unlv.edu
}

\begin{abstract}
This paper addresses two issues -- the time-inconsistency of optimal policy and the controllability of target variables within new-classical and new-Keynesian model structures. We can resolve both issues by delegation. That is, we design central bank loss functions by determining the two target values and the weight between the two targets. With a single decision maker, the time-inconsistency issue does not exist; the target controllability issue does. Delegating the long-run target values (target variables' equilibriums under the Ramsey optimal policy) and the same weight as society to the central bank can achieve Ramsey optimality and path controllability. With multiple decision makers (game), both issues of time-inconsistency and target controllability exist and the delegation becomes more complicated. The long-run target values can only achieve asymptotic, not path, controllability. Path controllability requires the delegation of short-run target values, which commits or binds the central bank to follow exactly the Ramsey optimal paths. The short-run inflation target value conforms to the macroeconomic structure (i.e., Phillips curve). With path controllability, the constant average and state-contingent inflation biases are removed. To eliminate the stabilization bias, the delegated weight must differ from society in a dynamic game. When the Phillips curve exhibits output (inflation) persistence, the central bank must place more weight on output (inflation) stabilization. When the Phillips curve exhibits principally forward-looking behavior, the delegated weight can require a conservative or liberal central bank. In sum, delegating certain short-run target values and a different weight can cause discretionary monetary policy to prove Ramsey optimal and path controllable in a dynamic game.
\end{abstract}

\section{J.E.L. Classification: $\quad$ E420, E520, E580}

Key words: $\quad$ Optimal Policy, Controllability, Policy Rules

* Professor Yuan gratefully acknowledges the financial support from the Ministry of Education of China. This paper is a research result of the Humanities and Social Science Project 10YJA790238.

** Corresponding author. 


\section{Introduction}

Monetary economists continue to debate two long-standing policy issues -- the timeinconsistency of optimal policy (e.g., see Kydland and Prescott, 1977 and Calvo, 1978) and target controllability (e.g., see Kalman, 1960; Tinbergen, 1952, 1963; Preston 1974; and Aoki, 1975). This paper tackles both issues, which usually receive separate treatment, making discretionary (time-consistent) policy Ramsey optimal and target controllable in dynamic models. $^{1}$

Researchers typically delegate a loss function to the central bank to solve the timeinconsistency of optimal policy, for instance, the conservative central banker of Rogoff (1985), the inflation contract of Walsh (1995), the employment contract of Chortareas and Miller (2003), the inflation target of Svensson (1997), the nominal income growth target of Beetsma and Jensen (1999) and Jensen (2002), the price-level target of Vestin (2006), and the consistent target of Yuan, et al. (2011), and so on. Though the various delegation schemes ostensibly differ and lead to different interpretations, many delegations prove identical in effect. ${ }^{2}$

The delegation approach solves the time-inconsistency problem in a straightforward fashion. Under the delegated loss function, the central bank operates monetary policy with discretion. As a result, monetary policy is time-consistent. The main issue, however, is whether discretionary policy can approximate or even reproduce the Ramsey policy.

\footnotetext{
${ }^{1}$ Yuan, et al. (2011) study both issues in static models.

${ }^{2}$ Yuan, et al. (2011) compare their designed loss function with the loss functions in Svensson (1997) without employment persistence, Walsh (1995), and Chortareas and Miller (2003), and show that the four loss functions generate identical results with respect to the policy decision. Svensson (1997) also observes that the inflationtarget-conservative loss function without employment persistence mimics the linear inflation contract in Walsh (1995).
} 
Brockett and Mesarović (1965) define three types of controllability (reproducibility):

point, path, and asymptotic controllability. ${ }^{3}$ Point controllability means that the policy makers can achieve certain target values at a specified point in time. In practice, policy makers probably desire more than point controllability, that is, the stronger notion of path controllability. Path controllability means that policy makers can make target variables follow some prescribed trajectories over a certain time interval (see Aoki, 1975). Obviously, path controllability implies point controllability, whereas the converse generally does not hold. Asymptotic controllability means that policy makers can reach the target values at infinity.

Path controllability plays a growing role in dynamic models of economic policy. ${ }^{4}$ To the best of our knowledge, the economics literature does not consider asymptotic controllability. This paper considers both path and asymptotic controllability, which prove useful concepts in describing the paths of target variables. ${ }^{5}$

Our analysis proceeds in several steps. First, given a social loss function, we determine the Ramsey optimal policy path. This optimal trajectory determines the design of the central bank loss function. Second, we design the central bank loss function by determining three key

3 Brockett and Mesarović (1965) use "reproducibility" instead of "controllability". The terminology "reproducibility" appears in the engineering literature, whereas the same concept of "controllability" appears in the economics literature. Also, they introduce four rather than three types: point, locally path, uniformly path, and asymptotic controllability. We here refer to both locally and uniformly path controllability as path controllability. In addition, the literature also calls path controllability, functional or perfect controllability.

${ }^{4}$ See, for example, Nyberg and Viotti (1978), Buiter and Gersovitz (1981, 1984), Wohltmann (1981, 1985), Tondini (1984), and Maas and Nijmeijer (1994). Besides extending Tinbergen's "static controllability" to dynamic controllability (Preston 1974), the literature extends controllability from one-decision-maker to multiple-decisionmakers (game) context. See Acocella and Di Bartolomeo (2006), Acocella et al. (2006, 2007), Hughes Hallett et al. (2010) for controllability in a game context.

5 Phillips (1954) considers the time paths of economic variables as well as the static analysis of the final equilibrium. That is, his analysis involves the idea of path and asymptotic controllability. 
parameters - the two target values (i.e., the inflation rate and output gap), and the weight between the two targets. Third, we determine the two target values. We find that the target variables' equilibriums under the Ramsey policy prove jointly asymptotically controllable. We call these equilibrium values the long-run target values. The discretionary policy with these long-run target values, if the system converges, can only achieve asymptotic rather than path controllability. Fourth, to obtain path controllability, we adopt state-contingent, short-run target values. Intuitively, the existence of time-inconsistency lures the central bank to deviate from the Ramsey optimal paths each period. As a result, we must delegate state-contingent, short-run target values each period. They commit and bind the central bank to follow exactly the Ramsey optimal paths. The short-run target values converge to the long-run target values in a step-by-step process. Also, they, though state-contingent, are predetermined and, thus, feasible in practice. With the short-run target values, discretionary policy proves path controllable and, thus, eliminates "the constant average and state-contingent inflation biases" (Svensson, 1997, p104). Fifth, when shocks occur, stabilization bias arises under discretionary policy. We can eliminate the stabilization bias by delegating to the central bank a deliberate weight, which differs from the social weight. The central bank with a different weight makes a different trade-off between the inflation rate and the output gap and, thus, stabilizes the shocks in exactly a Ramsey optimal fashion. In sum, delegation of state-contingent, short-run target values and the correct weight parameter cause discretionary policy to follow the Ramsey optimal path.

We determine the necessary and sufficient conditions for asymptotic controllability of constant target values. In contrast, the controllability literature generally considers the equations 
of motion, the available instruments, and the initial state of the system. We demonstrate that the constant target values, if the system converges, are jointly asymptotically controllable if and only if they satisfy the macroeconomic structure (i.e., the Phillips curve in our models) in equilibrium. The result holds under commitment and under discretion. We state our findings in Propositions 1 and 2. Intuitively, the macroeconomic structure (i.e., the Phillips curve) constrains the target variables and as a result, proper target values will exhibit the Phillips curve trade-off. The target variables' equilibriums under the Ramsey policy, as an example of constant target values, satisfy the Phillips curve in equilibrium and, therefore, are jointly asymptotically controllable.

The paper unfolds as follows. Section 2 determines the Ramsey optimal paths of target variables, using the social loss function with commitment, within new-classical and newKeynesian models. We illustrate the time-inconsistency and target uncontrollability of Ramsey optimal policy, which leads to Proposition 1. Section 3 extends Proposition 1 to Proposition 2. We design the central bank loss functions by determining the long-run and short-run target values as well as the weight parameter in the two models. Section 4 discusses two situations where the delegated weight equals the social weight. Section 5 concludes.

\section{Time-Inconsistency and Target Controllability of Ramsey Optimal Policies}

New-Classical Model with Output Persistence

The model follows Svensson (1997). We simply adopt it without much description. See Svensson (1997) for more details.

Society minimizes the following intertemporal loss function 


$$
\mathbb{L}=E_{0}\left(\sum_{t=1}^{\infty} \beta^{t-1} L_{t}\right),
$$

where $\beta(0<\beta<1)$ is the discount factor and $E$ is the expectations operator. The period loss function equals the following

$$
L_{t}=\frac{1}{2}\left[\left(\pi_{t}-\pi^{*}\right)^{2}+\lambda\left(x_{t}-x^{*}\right)^{2}\right]
$$

where $\pi$ is the inflation rate, $x$ is the output gap, $\pi^{*}$ is the socially desirable inflation rate, $x^{*}$ is the socially desirable output gap, and $\lambda$ is the social weight on output stabilization relative to inflation stabilization around their respective targets.

The economic structure includes an expectations-augmented Phillips curve with output persistence and rational expectations

$$
\begin{aligned}
& x_{t}=\eta x_{t-1}+\alpha\left(\pi_{t}-\pi_{t}^{e}\right)+u_{t}, \text { and } \\
& \pi_{t}^{e}=E_{t-1} \pi_{t},
\end{aligned}
$$

where $\eta(0 \leq \eta<1)$ measures the degree of output gap persistence, $\alpha$ is the response of output gap to unexpected inflation, $\pi_{t}^{e}$ denotes inflation expectations in period $t-1$ of the inflation rate in period $t$, and $u_{t}$ is an i.i.d. supply shock with mean 0 and variance $\sigma^{2}$.

Ramsey Optimal Policy. The central bank minimizes the social intertemporal loss function (1) with period loss function (2) subject to Eqs. (3) and (4). The socially optimal paths of target variables under commitment equal ${ }^{6}$

$$
\begin{aligned}
& \pi_{t}=\pi^{*}+d^{*} u_{t}, \text { and } \\
& x_{t}=\eta x_{t-1}+\left(1+\alpha d^{*}\right) u_{t}, \text { where }
\end{aligned}
$$

\footnotetext{
${ }^{6}$ See Svensson (1997).
} 


$$
d^{*}=-\frac{\lambda \alpha}{1-\beta \eta^{2}+\lambda \alpha^{2}}
$$

Time-Inconsistency of Ramsey Optimal Policy. Ramsey optimal policy equals an ex ante plan made by a social planner, who controls all instruments -- the instruments of the policy makers and the private sector. Such an ex ante plan, however, is not implementable. With multiple decision makers (game), policy makers cannot control other players' instruments, and implements policies using discretion. ${ }^{7}$ Discretionary policies are time-consistent, but nonoptimal.

Target Controllability of Ramsey Optimal Policy. In addition to time-inconsistency, Ramsey optimal policy still faces target controllability.

The Ramsey optimal paths of the target variables in Eqs. (5a) and (5b) consist of systematic and random components. We denote the systematic components as follows

$$
\left(\bar{\pi}_{t}, \bar{x}_{t}\right)=\left(\pi^{*}, \eta x_{t-1}\right)
$$

and random components or reactions to supply shocks as follows

$$
\left(\bar{\pi}_{t}\left(u_{t}\right), \bar{x}_{t}\left(u_{t}\right)\right)=\left(d^{*} u_{t},\left(1+\alpha d^{*}\right) u_{t}\right)
$$

Controllability is irrelevant to external shocks in our specific models with quadratic loss functions, linear Phillips curves, and additive shocks. Accordingly, we ignore shocks and consider the systematic components when we consider controllability.

The systematic components converge to $\left(\pi^{*}, 0\right)$

$$
(\bar{\pi}, \bar{x}) \equiv \lim _{t \rightarrow \infty}\left(\bar{\pi}_{t}, \bar{x}_{t}\right)=\left(\pi^{*}, 0\right)
$$

\footnotetext{
${ }^{7}$ For discretionary policy, refer to Svensson (1997).
} 
where $\bar{\pi}\left(=\pi^{*}\right)$ and $\bar{x} \quad(=0)$ denote the equilibriums (limits) of the inflation rate and the output gap, respectively.

In addition, the convergence persistence of the inflation rate and the output gap equals 0 and $\eta$, respectively. That is, the inflation rate hits its equilibrium, $\bar{\pi}=\pi^{*}$, immediately with zero persistence, and the output gap converges to its equilibrium, $\bar{x}=0$, with persistence $\eta$.

Consider target controllability. Obviously, the policy maker can point control the inflation rate each period $\left(\bar{\pi}_{t}=\pi^{*}\right)$ and, thus, control its path. The policy maker cannot point control the output gap each period $\left(\bar{x}_{t} \neq x^{*}\right)$ and cannot even asymptotically control the output gap $\left(\bar{x} \neq x^{*}\right.$, if $x^{*} \neq 0$ ). To understand why the policy maker cannot asymptotically control the social output gap target value, if $x^{*} \neq 0$, consider the Phillips curve in Eq. (3) and the rational expectations assumption in Eq. (4). In equilibrium, ignoring shocks, $\pi_{t}^{e}=E_{t-1} \bar{\pi}=\bar{\pi}, \pi_{t}=\bar{\pi}$, and the Phillips curve equals to

$$
\bar{x}=\eta \bar{x} \text {, i.e., } \bar{x}=0 .
$$

That is, the output gap must equal zero in equilibrium. If we require asymptotic controllability of the output gap (i.e., $\bar{x}=x^{*}$ ), then we must observe

$$
x^{*}=0 .
$$

On the contrary, if $x^{*}=0$, then the policy maker can asymptotically control the zero target value because $\bar{x}=0=x^{*}$. In sum, Eq. (11) is the necessary and sufficient condition for asymptotic controllability of the output gap target value, $x^{*} .8$

\footnotetext{
${ }^{8}$ In the simplicity of the new-classical model, the asymptotic controllability condition in Eq. (11) proves trivial. This condition is nontrivial, however, in the new-Keynesian model.
} 
The target variables' equilibriums, $\bar{\pi}\left(=\pi^{*}\right)$ and $\bar{x}(=0)$, satisfy the necessary and sufficient condition and, thus, are jointly asymptotically controllable. They are the long-run target values according to the definition in the introduction.

\section{A Hybrid New-Keynesian Model}

Researchers developed and applied new-Keynesian models in the past decade. We adopt a hybrid new-Keynesian model, which combines the possibility of forward- and backward-looking inflation. This model reduces to a purely forward-looking or a purely backward-looking model by choosing the extreme values of the parameter that indexes expectations across the forwardand backward-looking dimensions. See Clarida et al. (1999) for more details.

The social intertemporal loss function equals ${ }^{9}$

$$
\mathbb{L}=E_{0}\left(\sum_{t=0}^{\infty} \beta^{t} L_{t}\right)
$$

with the same period loss function as in Eq. (2).

Aggregate supply equals an expectations-augmented Phillips curve with forward-looking expectations and endogenous inflation

$$
\pi_{t}=\kappa X_{t}+\phi \pi_{t-1}+(1-\phi) \beta E_{t} \pi_{t+1}+u_{t}
$$

where $\pi, x$, and $\beta$ are defined as before, $\kappa(\kappa>0)$ is the sensitivity of the inflation rate to the output gap, $\phi$ indexes the degree of lagged versus expected future inflation rates, $u_{t}$ is a cost-push shock that follows an $\mathrm{AR}(1)$ process

\footnotetext{
9 The expectation operator, $E_{t}(\cdot)$, slightly differs from that in the new-classical model in that the private sector forms rational expectations in the new-Keynesian model at the beginning of the present period and in the new-classical model from the previous period. We just follow the usual definitions in literature for convenience.
} 


$$
u_{t}=\rho u_{t-1}+\hat{u}_{t},
$$

where $0 \leq \rho<1$, and $\hat{u}_{t}$ is a white noise residual.

We do not introduce aggregate demand (IS curve), which involves a nominal interest rate, the policy instrument. Once we determine the optimal paths for $\left\{\pi_{t}, x_{t}\right\}_{t=0}^{\infty}$ using the social loss function and the Phillips curve, both of which do not involve the interest rate, then we can pin down the optimal path of interest rates through the IS curve. So the Phillips curve proves critical for policy.

Ramsey Optimal Policy. The consolidated first-order condition of optimal policy under the social intertemporal loss function (12) with period loss function (2) subject to the Phillips curve in Eq. (13) equals ${ }^{10}$

$$
\begin{aligned}
& \left(x_{0}-x^{*}\right)-\phi \beta\left(E_{0} x_{1}-x^{*}\right)=-\frac{\kappa}{\lambda}\left(\pi_{0}-\pi^{*}\right) \quad \text { for } t=0, \text { and } \\
& \left(E_{0} x_{t}-x^{*}\right)-\phi \beta\left(E_{0} x_{t+1}-x^{*}\right)-(1-\phi)\left(E_{0} x_{t-1}-x^{*}\right)=-\frac{\kappa}{\lambda}\left(E_{0} \pi_{t}-\pi^{*}\right) \quad \text { for } t \geq 1 .
\end{aligned}
$$

Combining the first-order conditions (15a) and (15b) and the Phillips curve (Eq. 13) leads to Ramsey optimal paths of target variables ${ }^{11}$

$$
\begin{array}{ll}
\left(\pi_{0}-\bar{\pi}\right)=\delta_{\pi 0}\left[\phi\left(\pi_{-1}-\bar{\pi}\right)+(1-\phi) \kappa\left(x^{*}-\bar{x}\right)\right]+h_{0} u_{0} & \text { for } t=0, \\
\left(x_{0}-\bar{x}\right)=\delta_{x 0}\left[\left(x^{*}-\bar{x}\right)+\frac{\phi \kappa\left(\pi^{*}-\pi_{-1}\right)}{\lambda\left(1-a-\phi^{2} \beta\right)}\right]+f_{0} u_{0} & \text { for } t=0, \\
\left(\pi_{t}-\bar{\pi}\right)=\delta\left(\pi_{t-1}-\bar{\pi}\right)+\frac{\delta \rho}{\delta \rho d-a}\left[(1-\phi \beta \rho) u_{t}-(1-\phi) u_{t-1}\right], & \text { or } \\
\left(\pi_{t}-\bar{\pi}\right)=\delta\left(\pi_{t-1}-\bar{\pi}\right)+\frac{\delta \rho}{\delta \rho d-a}\left[\begin{array}{l}
\left.\left(\phi-\phi_{c}\right)\left(1-\beta \rho^{2}\right) u_{t-1}\right] \\
+(1-\phi \beta \rho) \hat{u}_{t}
\end{array}\right] \quad \text { for } t \geq 1 ; \text { and }
\end{array}
$$

\footnotetext{
${ }^{10}$ See Eqs. (A.3a) and (A.3b) in Appendix A.

${ }^{11}$ See Eqs. (A.14), (A.24), (A.35) and (A.39) and their derivation in Appendix A.
} 


$$
\left(x_{t}-\bar{x}\right)=\delta\left(x_{t-1}-\bar{x}\right)-\frac{\kappa \delta \rho}{\lambda(\delta \rho d-a)} u_{t} \quad \quad \text { for } t \geq 1,
$$

where $\delta_{\pi 0}, \delta_{x 0}, h_{0}, f_{0}$ are defined in Appendix A, and $\delta$ is a root of the characteristic equation

$$
\begin{aligned}
& a \beta^{2} \delta^{4}-\beta \delta^{3}+b \delta^{2}-\delta+a=0 \text { with } \\
& a \equiv \phi(1-\phi), \\
& b \equiv 1+\frac{\kappa^{2}}{\lambda}+\phi^{2} \beta+(1-\phi)^{2} \beta=1+\beta+\frac{\kappa^{2}}{\lambda}-2 a \beta, \\
& d \equiv a \beta^{2}\left(\delta^{2}+\delta \rho+\rho^{2}\right)-\beta(\delta+\rho)+b, \\
& \bar{\pi} \equiv \frac{\kappa\left[\kappa \pi^{*}+\lambda \phi(1-\beta) x^{*}\right]}{\kappa^{2}+\lambda a(1-\beta)^{2}}, \\
& \bar{x} \equiv \frac{(1-\phi)(1-\beta)}{\kappa} \bar{\pi}, \text { and } \\
& \phi_{c} \equiv \frac{1-\rho}{1-\beta \rho^{2}}, \text { and } 0<\phi_{c} \leq 1 .
\end{aligned}
$$

For the purely forward-looking, new-Keynesian model $(\phi=0)$, the solution in Eqs. (17a) and (17b) reduces to the solution in Clarida et al. (1999, p1703-1704) by noting that ${ }^{12}$

$$
\frac{1}{d}=\frac{\delta}{1-\delta \beta \rho} \text {, when } \phi=0 \text { and } 1
$$

Eqs. (18a), (18b), and (18c) determine, what we call, the system convergence persistence, $\delta$. We cannot determine whether a root $\delta \in(0,1)$ exists for any $\phi \in(0,1)$. But for the two extreme cases of $\phi=0$ and $\phi=1$, the characteristic Eq. (18a) reduces to $\beta \delta^{2}-b \delta+1=0$, and a root $\delta \in(0,1)$ does exist. That is, the system can converge under the Ramsey policy for the two

\footnotetext{
12 If $\phi=0$ or 1 , then $a=0, d=b-\beta(\delta+\rho), d \delta=b \delta-\beta \delta^{2}-\delta \beta \rho$, and the characteristic equation reduces to $\beta \delta^{2}-b \delta+1=0$. Thus, $b \delta-\beta \delta^{2}=1$. Therefore, $d \delta=1-\delta \beta \rho$ or $1 / d=\delta /(1-\delta \beta \rho)$.
} 
extreme cases. Whether a root $\delta \in(0,1)$ exists, $\delta$ depends on the model structural parameters (i.e., $\phi, \beta, \kappa$, and $\lambda$ ), and does not relate to the persistence of the cost-push shock. We attribute the irrelevance of the cost-push shocks to the quadratic loss function and the linear Phillips curve with additive shocks.

The critical value, $\phi_{c}$, is meaningful. For convenience, we define the Phillips curve as principally forward-looking (backward-looking) if $0 \leq \phi \leq \phi_{c} \quad\left(\phi_{c} \leq \phi \leq 1\right)$. Whether the Phillips curve exhibits principally forward- or backward-looking behavior results in different (negative or positive) responses of the inflation rate to cost-push shocks and, we will see in next section, different short-run target values and weight parameter that are delegated to the central bank.

The critical value $\phi_{c}$ depends on both the discount factor $\beta$ and the cost-push shock persistence $\rho$, and responds to them as follows ${ }^{13}$

$$
\begin{aligned}
& \partial \phi_{c} / \partial \beta>0, \text { and } \\
& \partial \phi_{c} / \partial \rho<0 .
\end{aligned}
$$

That is, a more important future and/or less persistent cost-push shocks lead to a higher critical value and, thus, the inflation rate for a given inflation rate persistence $\phi$ more likely exhibits principally forward-looking behavior $\left(\phi \leq \phi_{c}\right)$.

Accordingly, whether the Phillips curve exhibits principally forward- or backwardlooking behavior depends on the three parameters, $\phi, \beta$, and $\rho$, rather than merely $\phi$. We refer to the parameters, $\phi, \beta$, and $\rho$, as dynamic parameters, since setting them equal to zero produces a

$13 \frac{\partial \phi_{c}}{\partial \beta}=\frac{\rho^{2}(1-\rho)}{\left(1-\beta \rho^{2}\right)^{2}}>0$ and $\frac{\partial \phi_{c}}{\partial \rho}=-\frac{(1-\beta)+\beta(1-\rho)^{2}}{\left(1-\beta \rho^{2}\right)^{2}}<0$ for $0<\rho<1$. 
static model. As such, the dynamic parameters are $\beta$ and $\eta$ in the new-classical model, where supply shocks equal white noise. We will see that the delegated weight depends on dynamic parameters and/or the system convergence persistence in a dynamic game model and equals the social weight in a static model.

Time-Inconsistency of Ramsey Optimal Policy. The first-order conditions (15a) and (15b) suggest the time-inconsistency of the Ramsey optimal solution. Ramsey optimal policy requires that the present period follows condition (15a) and that future periods follow condition (15b). In practice, however, the central bank re-minimizes the loss function each period and, thus, always follows the first-order condition (15a).

Target Controllability of Timeless Perspective Optimal Policy. We adopt optimality from the timeless perspective, ${ }^{14}$ and analyze Eqs. (17a) and (17b) for $t \geq 1$.

The optimal paths of the target variables in Eqs. (17a) and (17b) consist of systematic components denoted as

$$
\left(\bar{\pi}_{t}, \bar{x}_{t}\right)=\left(\bar{\pi}+\delta\left(\pi_{t-1}-\bar{\pi}\right), \bar{x}+\delta\left(x_{t-1}-\bar{x}\right)\right)
$$

and random components related to cost-push shocks denoted as

$$
\left(\bar{\pi}_{t}\left(u_{t}\right), \bar{x}_{t}\left(u_{t}\right)\right)=\left(\frac{\delta \rho}{\delta \rho d-a}\left[(1-\phi \beta \rho) u_{t}-(1-\phi) u_{t-1}\right],-\frac{\kappa \delta \rho}{\lambda(\delta \rho d-a)} u_{t}\right) .
$$

Similarly, controllability does not depend on external shocks, and we consider the systematic components. The systematic components converge to $(\bar{\pi}, \bar{x})$, if $\delta<1$,

$$
(\bar{\pi}, \bar{x})=\lim _{t \rightarrow \infty}\left(\bar{\pi}_{t}, \bar{x}_{t}\right)
$$

\footnotetext{
${ }^{14}$ Woodford (1999) introduces the concept of optimality from a "timeless perspective," which means the policy the central bank "to which it would have wished to commit itself to at a date far in the past." (293, italics in original).
} 
where Eqs. (18e) and (18f) define $\bar{\pi}$ and $\bar{x}$. So $\bar{\pi}$ and $\bar{x}$ are the equilibriums (limits) of the inflation rate and the output gap. The two target variables evolve to their respective equilibriums with the same system convergence persistence, $\delta$.

Now, consider target controllability. The policy makers cannot point control both the inflation rate and the output gap each period, since $\bar{\pi}_{t} \neq \pi^{*}$ and $\bar{x}_{t} \neq x^{*}$. They also generally cannot asymptotically control the targets, since $\bar{\pi} \neq \pi^{*}$ and $\bar{x} \neq x^{*} .{ }^{15}$

Consider Eq. (18f). The equilibriums of the target variables satisfy the Phillips curve in equilibrium. That is,

$$
\bar{\pi}=\kappa \bar{x}+\phi \bar{\pi}+(1-\phi) \beta \bar{\pi} .
$$

The two targets are jointly asymptotically controllable, if the two target values, $\pi^{*}$ and $x^{*}$, satisfy the Phillips curve in equilibrium. That is,

$$
\pi^{*}=\kappa x^{*}+\phi \pi^{*}+(1-\phi) \beta \pi^{*}
$$

We can easily verify this result. Rearranging Eq. (25) gives

$$
x^{*}=\frac{(1-\phi)(1-\beta)}{\kappa} \pi^{*}
$$

Substituting Eq. (26) into Eq. (18e) generates

$$
\bar{\pi} \equiv \frac{\kappa\left[\kappa \pi^{*}+\lambda \phi(1-\beta) x^{*}\right]}{\kappa^{2}+\lambda a(1-\beta)^{2}}=\frac{\kappa\left[\kappa \pi^{*}+\lambda \phi(1-\beta) \frac{(1-\phi)(1-\beta)}{\kappa} \pi^{*}\right]}{\kappa^{2}+\lambda a(1-\beta)^{2}}=\pi^{*} .
$$

Using Eqs. (18f), (27), and (26) in sequence produces

${ }^{15}$ If $\phi=0$, then the policy makers can asymptotically control the inflation rate $\left(\bar{\pi}=\pi^{*}\right)$. 


$$
\bar{x} \equiv \frac{(1-\phi)(1-\beta)}{\kappa} \bar{\pi}=\frac{(1-\phi)(1-\beta)}{\kappa} \pi^{*}=x^{*} .
$$

That is, the two target values, $\pi^{*}$ and $x^{*}$, are asymptotically controllable. Conversely, if the two target values, $\pi^{*}$ and $x^{*}$, are asymptotically controllable (joint asymptotic controllability implies $\bar{\pi}=\pi^{*}$ and $\left.\bar{x}=x^{*}\right)$, then they satisfy Eq. (25).

In sum, Eq. (25) provides the necessary and sufficient condition for joint asymptotic controllability of the social target values, $\pi^{*}$ and $x^{*}$. The target variables' equilibriums, $\bar{\pi}$ and $\bar{X}$ defined in Eqs. (18e) and (18f), satisfy the necessary and sufficient condition and, thus, are jointly asymptotically controllable. They are the long-run target values according to the definition in the introduction.

\section{Joint Asymptotic Controllability}

The controllability literature usually considers the equations of motion, the available instruments, and the initial state of the system. Rather, we find the necessary and sufficient condition for the joint asymptotic controllability of constant target values. We summarize the above results in Proposition 1.

Proposition 1. If the system converges under the Ramsey policy with period social loss function in Eq. (2) subject to a linear Phillips curve, $\pi^{*}$ and $x^{*}$ are jointly asymptotically controllable if and only if they satisfy the Phillips curve in equilibrium.

Proof. We prove the proposition in a more simple way. Take the hybrid new-Keynesian model as an example.

Sufficiency. If $\pi^{*}$ and $x^{*}$ satisfy the Phillips curve in Eq. (25), then combining Eq. (25) 
and Eq. (13) produces the Phillips curve around its equilibrium as follows

$$
\left(\pi_{t}-\pi^{*}\right)=\kappa\left(x_{t}-x^{*}\right)+\phi\left(\pi_{t-1}-\pi^{*}\right)+(1-\phi) \beta\left(E_{t} \pi_{t+1}-\pi^{*}\right)+u_{t}
$$

Now, the optimization problem equals the minimization of the social intertemporal loss function (12) with period loss function (2) subject to the Phillips curve (29). Obviously, the optimal paths $\left(\pi_{t}-\pi^{*}, x_{t}-x^{*}\right)$ will converge to $(0,0)$, if the system converges. That is, $\left(\pi_{t}, x_{t}\right)$ converge to $\left(\pi^{*}, x^{*}\right)$. Therefore, $\pi^{*}$ and $x^{*}$ are jointly asymptotically controllable.

Necessity. If $\pi^{*}$ and $x^{*}$ are jointly asymptotically controllable, $\bar{\pi}=\pi^{*}$ and $\bar{x}=x^{*}$ hold. The Phillips curve in equilibrium, which involves $\bar{\pi}$ and $\bar{x}$, leads to the condition that $\pi^{*}$ and $x^{*}$ satisfy the Phillips curve in equilibrium.

\section{Designing Central Bank Loss Functions}

Though we determine the necessary and sufficient condition for jointly asymptotically controllable target values, the social target values generally do not satisfy this condition because the social loss function (e.g., the representative household's utility, Arrow's (1951) social welfare function, or Rawls's (1971) maximin criterion) reflects a normative problem in philosophy and usually does not relate to economic models.

Proposition 1, however, provides a way to design the central bank loss function. We extend Proposition 1 to discretionary policy under a central bank loss function. As noted in the introduction, we design the central bank loss function by determining the three key parameters -

the two target values of the inflation rate and the output gap denoted as $\left(\pi^{b}, x^{b}\right)$ and the weight on output stabilization relative to inflation stabilization denoted as $\lambda^{b}$. Thus, the central bank period loss function equals 


$$
L_{t}^{b}=\frac{1}{2}\left[\left(\pi_{t}-\pi^{b}\right)^{2}+\lambda^{b}\left(x_{t}-x^{b}\right)^{2}\right]
$$

Proposition 2. If the system converges under the discretionary policy with period central bank loss function in Eq. (30) subject to a linear Phillips curve, $\pi^{b}$ and $x^{b}$ are jointly asymptotically controllable if and only if they satisfy the Phillips curve in equilibrium.

The proof is similar to Proposition 1 by noting that the difference between Ramsey policy and discretionary policy merely reflects the different sequence of optimizations of decision makers.

For convenience, denote the equilibriums of the inflation rate and the output gap under the discretionary policy with the central bank loss function, respectively, as $\tilde{\pi}$ and $\tilde{x}$, which are the counterparts of $\bar{\pi}$ and $\bar{x}$, the equilibriums under the Ramsey policy with the social loss function. On the one hand, we require that the discretionary policy is Ramsey optimal (i.e., at least $\tilde{\pi}=\bar{\pi}$ and $\tilde{x}=\bar{x}$ ); on the other hand, we require that the constant target values are asymptotically controllable (i.e., $\tilde{\pi}=\pi^{b}$ and $\tilde{x}=x^{b}$ ). As a result,

$$
\left(\pi^{b}, x^{b}\right)=(\bar{\pi}, \bar{x})
$$

Since $\bar{\pi}$ and $\bar{x}$ satisfy the Phillips curve in equilibrium, $\bar{\pi}$ and $\bar{x}$ are jointly asymptotically controllable under the discretionary policy if the system converges. Thus, $\bar{\pi}$ and $\bar{x}$ are also the long-run target values under the discretionary policy.

We will see that the discretionary policy with the long-run target values, however, is generally not path controllable in the two example models, where the desired trajectories equal their respective Ramsey optimal paths. Path uncontrollability leads to social losses. The loss function in Eq. (30) still needs improvement. 
The long-run target values cannot achieve path controllability (or point controllability each period). Intuitively, the existence of time-inconsistency lures the central bank to deviate from the Ramsey optimal paths each period. As a result, we must delegate short-run target values each period. They commit and bind the central bank to follow exactly the Ramsey optimal paths.

Path controllability requires that $\left(\tilde{\pi}_{t}, \tilde{x}_{t}\right)$, which denote the systematic paths of the inflation rate and the output gap under the discretionary policy with the designed loss function, replicate $\left(\bar{\pi}_{t}, \bar{x}_{t}\right)$ for each period $t$. At the same time, we require that the short-run target values, denoted as $\pi_{t}^{b}$ and $x_{t}^{b}$, are controllable. That is, $\left(\tilde{\pi}_{t}, \tilde{x}_{t}\right)=\left(\pi_{t}^{b}, x_{t}^{b}\right)$. As a result,

$$
\left(\pi_{t}^{b}, x_{t}^{b}\right)=\left(\bar{\pi}_{t}, \bar{x}_{t}\right)
$$

The corresponding loss function equals

$$
L_{t}^{b}=\frac{1}{2}\left[\left(\pi_{t}-\pi_{t}^{b}\right)^{2}+\lambda^{b}\left(x_{t}-x_{t}^{b}\right)^{2}\right] .
$$

Though the short-run target values are state-contingent, they are predetermined because $\bar{\pi}_{t}\left(\bar{x}_{t}\right)$ is the weighted average of lagged values, $\pi_{t-1}\left(x_{t-1}\right)$, and long-run target values, $\bar{\pi}$ $(\bar{x})$, and the weight on $\pi_{t-1}\left(x_{t-1}\right)$ equals the convergence persistence under the Ramsey optimal policy. The predetermined target values are feasible in practice.

With the short-run target values, discretionary policy is path controllable and, thus, eliminates the constant average and state-contingent inflation biases. This leaves the stabilization bias. We can eliminate this bias by determining a proper weight, $\lambda^{b}$, such that

$$
\left(\tilde{\pi}\left(u_{t}\right), \tilde{x}_{t}\left(u_{t}\right)\right)=\left(\bar{\pi}\left(u_{t}\right), \bar{x}_{t}\left(u_{t}\right)\right),
$$

where $\left(\tilde{\pi}\left(u_{t}\right), \tilde{x}_{t}\left(u_{t}\right)\right)$ denote the reactions to shocks under the discretionary policy with the designed loss function. Actually, we pin down the weight through one of the two equations, 
$\tilde{\pi}\left(u_{t}\right)=\bar{\pi}\left(u_{t}\right)$ or $\tilde{x}_{t}\left(u_{t}\right)=\bar{x}_{t}\left(u_{t}\right)$. Once one equation holds, the other equation also holds because the inflation rate and the output gap are linked through the Phillips curve.

Now, we design central bank loss functions using the above approach for the two different models.

Designing the Central Bank Loss Function in the New-Classical Model with Output Persistence Asymptotic Controllability under Discretion with the Long-Run Target Values. By Eqs. (9) and

$$
\pi^{b}=\bar{\pi}=\pi^{*}, \text { and } x^{b}=\bar{x}=0
$$

The corresponding central bank period loss function equals

$$
L_{t}^{b}=\frac{1}{2}\left[\left(\pi_{t}-\pi^{*}\right)^{2}+\lambda^{b} x_{t}^{2}\right]
$$

The outcomes under the discretionary policy with period loss function (36) equal ${ }^{16}$

$$
\tilde{d}=-\frac{\lambda^{b} \alpha+\beta \alpha c^{2}}{1-\beta \eta^{2}+\lambda^{b} \alpha^{2}+\beta \alpha^{2} c^{2}}
$$

Denote the systematic paths as

$$
\left(\tilde{\pi}_{t}, \tilde{x}_{t}\right)=\left(\pi^{*}-c x_{t-1}, \eta x_{t-1}\right)
$$

Obviously,

\footnotetext{
${ }^{16}$ See the discretionary policy in Svensson (1997). Our notation differs slightly from his. Setting the employment gap (output gap) target value to zero and replacing $\lambda$ with $\lambda^{b}$ and $\rho$ with $\eta$ creates the results in Eqs. (37) and (38).
} 


$$
(\tilde{\pi}, \tilde{x}) \equiv \lim _{t \rightarrow \infty}\left(\tilde{\pi}_{t}, \tilde{x}_{t}\right)=\left(\pi^{*}, 0\right) .
$$

That is, $\pi^{*}$ and 0 are jointly asymptotically controllable under the discretionary policy.

By Eqs. (7) and (39), generally

$$
\left(\tilde{\pi}_{t}, \tilde{x}_{t}\right) \neq\left(\bar{\pi}_{t}, \bar{x}_{t}\right) .
$$

That is, the discretionary policy is path uncontrollable, where the desired trajectories equal the Ramsey optimal paths.

Path Controllability with the State-Contingent, Short-Run Target Values. By Eqs. (7) and (32),

$$
\pi_{t}^{b}=\bar{\pi}_{t}=\pi^{*}, \text { and } x_{t}^{b}=\bar{x}_{t}=\eta x_{t-1} .
$$

The corresponding central bank period loss function equals

$$
L_{t}^{b}=\frac{1}{2}\left[\left(\pi_{t}-\pi^{*}\right)^{2}+\lambda^{b}\left(x_{t}-\eta x_{t-1}\right)^{2}\right] .
$$

Intuitively, with the short-run natural output gap $\eta x_{t-1}$ as the output gap target, the central bank does not possess an incentive to produce surprise inflation $\left(\pi_{t}-\pi_{t}^{e}\right)$ to raise the output gap above the short-run natural gap $\eta x_{t-1}$. As a result, the central bank can jointly realize the target values $\pi^{*}$ and $\eta x_{t-1}$. The formal calculation of discretionary policy with the target values verifies the intuition. The discretionary outcomes equal ${ }^{17}$

$$
\begin{aligned}
& \pi_{t}=\pi^{*}+\tilde{d} u_{t}, \text { and } \\
& x_{t}=\eta x_{t-1}+(1+\alpha \tilde{d}) u_{t},
\end{aligned}
$$

where with the same notation $\tilde{d}$ without causing confusion

$$
\tilde{d}=-\frac{\lambda^{b} \alpha}{1+\lambda^{b} \alpha^{2}} .
$$

${ }^{17}$ See Eqs. (B.11a) and (B.11b) and their derivation in Appendix B. Multiple equilibriums exist. We report one here. 
Denote the systematic paths and the reaction paths respectively as

$$
\begin{aligned}
& \left(\tilde{\pi}_{t}, \tilde{x}_{t}\right)=\left(\pi^{*}, \eta x_{t-1}\right), \text { and } \\
& \left(\tilde{\pi}_{t}\left(u_{t}\right), \tilde{x}_{t}\left(u_{t}\right)\right)=\left(\tilde{d} u_{t},(1+\alpha \tilde{d}) u_{t}\right) . \text { Thus, } \\
& \left(\tilde{\pi}_{t}, \tilde{x}_{t}\right)=\left(\bar{\pi}_{t}, \bar{x}_{t}\right)
\end{aligned}
$$

which means path controllability and, thus, eliminates the constant average and state-contingent inflation biases.

Elimination of Stabilization Bias. To eliminate stabilization bias, we set $\tilde{\pi}_{t}\left(u_{t}\right)=\bar{\pi}_{t}\left(u_{t}\right)$. That is, $\tilde{d}=d^{*}$, which produces ${ }^{18}$

$$
\lambda^{b}=\frac{1}{1-\beta \eta^{2}} \lambda
$$

The coefficient of the central bank's weight depends only on the dynamic parameters, $\beta$ and $\eta .{ }^{19}$ If $\beta$ and/or $\eta$ equal zero (i.e., the model is static), the central bank must exhibit the same weight as society; ${ }^{20}$ otherwise, it must exhibit weight-liberal behavior, ${ }^{21}$ contrary to the usual recommendation of appointing a (weight-) conservative central banker. Intuitively, since the output gap persists, a current output gap deviation from its target value will persist into the future and, thus, cause losses. To reduce losses, the central bank must place more weight on the output gap target (i.e., $\left.\lambda^{b}>\lambda\right)$.

\footnotetext{
${ }^{18}$ Yuan and Miller (2010) also obtain the result in Eq. (49).

${ }^{19}$ Here, we assume that supply shocks equal white noise. Thus, the weight does not reflect the characteristic of the supply shocks. We discuss the persistence of shocks in the new-Keynesian model.

${ }^{20}$ Yuan, et al. (2011) “...attribute the identical weight to the static models and/or the white-noise shocks. Policy makers do not face a trade-off between targets and/or between periods if the government delegates the correct target values to the central bank in static models with white-noise shocks." (p. 84).

${ }^{21}$ We will see that a weight-liberal or weight-conservative central banker may emerge under certain circumstances in the new-Keynesian model.
} 
In sum, the discretionary policy proves path controllable (removing the constant average and state-contingent inflation biases), and eliminates stabilization bias under the loss function in Eq. (43) with $\lambda^{b}$ defined in Eq. (49), resulting in Ramsey optimality.

\section{Designing Central Bank Loss Functions in a Hybrid New-Keynesian Model}

Divergence/Convergence under Discretion with the Long-Run Target Values. By Eq. (31),

$$
\pi^{b}=\bar{\pi}, \text { and } x^{b}=\bar{x}
$$

where $\bar{\pi}$ and $\bar{x}$ are defined in Eqs. (18e) and (18f). The corresponding central bank period loss function equals

$$
L_{t}^{b}=\frac{1}{2}\left[\left(\pi_{t}-\bar{\pi}\right)^{2}+\lambda^{b}\left(x_{t}-\bar{x}\right)^{2}\right] .
$$

The outcomes under discretionary policy with period loss function (51) equal ${ }^{22}$

$$
\begin{aligned}
& \left(x_{t}-\bar{x}\right)=\tilde{\delta}\left(x_{t-1}-\bar{x}\right)+f u_{t}, \text { and } \\
& \left(\pi_{t}-\bar{\pi}\right)=\tilde{\delta}\left(\pi_{t-1}-\bar{\pi}\right)-\frac{\lambda^{b}}{\kappa} f(1-\phi \beta \rho) u_{t},
\end{aligned}
$$

where $\tilde{\delta}$ is a root of the characteristic equation

$$
\begin{aligned}
& a \beta^{2} \tilde{\delta}^{3}-\beta \tilde{\delta}^{2}+\left(1+\frac{\kappa^{2}}{\lambda^{b}}+\phi^{2} \beta\right) \tilde{\delta}-\phi=0, \text { and } \\
& f=\frac{\tilde{\delta} \kappa}{\lambda^{b}\{[1-a \beta(\tilde{\delta}+\rho)] \tilde{\delta} \beta \rho-\phi\}} .
\end{aligned}
$$

Does a less-than-one root exist for the characteristic equation (53a)? Consider the two extreme cases of $\phi=0$ and $\phi=1$. For the case $\phi=0$, the characteristic equation (53a) reduces to $\beta \tilde{\delta}^{2}-\left(1+\kappa^{2} / \lambda^{b}\right) \tilde{\delta}=0$, and its two roots are $\tilde{\delta}=0$, which is a degenerative solution, and

\footnotetext{
${ }^{22}$ See Eqs. (C.9), (C.13), (C.14) and (C.16) and their derivation in Appendix C.
} 
$\tilde{\delta}=\left(1+\kappa^{2} / \lambda^{b}\right) / \beta>1$. The system diverges under the discretionary policy when $\phi=0$, in contrast to converging under the Ramsey policy. For the case $\phi=1$, the characteristic equation (53a) reduces to

$$
\beta \tilde{\delta}^{2}-\left(1+\frac{\kappa^{2}}{\lambda^{b}}+\beta\right) \tilde{\delta}+1=0
$$

We can show that the bigger root exceeds one and the smaller root lies between zero and one. The system can converge under discretion when $\phi=1$. Actually, time-inconsistency does not exist in the case $\phi=1$, and we discuss this special case further in the next subsection. When the lag index $\phi$ goes from 0 to 1 , the system changes from divergence to convergence. The system may also go through divergence, convergence, divergence, convergence, and so on. For the case $0<\phi<1$, we can conclude that $\bar{\pi}$ and $\bar{x}$ are jointly asymptotically controllable, if the characteristic equation (53a) does have a root that less than one, $\tilde{\delta}<1$.

Generally, the system is not path controllable under the discretionary policy with the long-run target values since $\tilde{\delta} \neq \delta$ where $\tilde{\delta}$ and $\delta$ are determined respectively by Eqs. (53a) and (18a).

Path Controllability with the Long-Run Target Values When $\phi=1$. For the case $\phi=1$, we obtain an interesting result. That is, when the Phillips curve in Eq. (13) exhibits purely backward-looking behavior with no expectations

$$
\pi_{t}=\kappa X_{t}+\pi_{t-1}+u_{t}
$$

we delegate to the central bank a simple loss function with the long-run target values ( $\pi^{b}=\bar{\pi}$ and $\left.x^{b}=\bar{x}\right)$ and the same weight $\left(\lambda^{b}=\lambda\right)$ as follows 


$$
L_{t}^{b}=\frac{1}{2}\left[\left(\pi_{t}-\bar{\pi}\right)^{2}+\lambda\left(x_{t}-\bar{x}\right)^{2}\right] .
$$

With the simple loss function (Eq. 56), the discretionary policy proves path controllable and eliminates stabilization bias!

When the Phillips curve equals Eq. (55), which does not involve expectations, the optimal problem reduces to a control theory, rather than game theory, problem. That is, only one decision maker exists rather than multiple decision makers. Time-inconsistency does not exist. We demonstrate this through the consolidated first-order conditions under the Ramsey optimal policy. The condition for $t=0$ (Eq. 15a) equals the condition for $t \geq 1$ (Eq. 15b), when $\phi=1$. That is, the Ramsey policy is time-consistent. As a result, we do not need to delegate to the central bank the short-run target values, which commit or bind central bank behavior. In addition, the stabilization bias does not exist either. Accordingly, the central bank adopts the same weight $\left(\lambda^{b}=\lambda\right)$. When $\lambda^{b}=\lambda^{23}$

$$
\begin{aligned}
& \tilde{\delta}=\delta \Leftrightarrow \phi=1, \text { and } \\
& \left(\tilde{\pi}_{t}\left(u_{t}\right), \tilde{x}_{t}\left(u_{t}\right)\right)=\left(\bar{\pi}_{t}\left(u_{t}\right), \bar{x}_{t}\left(u_{t}\right)\right) .
\end{aligned}
$$

That is, the policy under the delegation of the long-run target values $\left(\pi^{b}=\bar{\pi}\right.$ and $\left.x^{b}=\bar{x}\right)$ and the same weight $\left(\lambda^{b}=\lambda\right)$ proves path controllable and of no stabilization bias, when $\phi=1$.

Though time-inconsistency does not exist, the issue of target controllability does. The target variables' equilibriums in Eqs. (18e) and (18f) with $\phi=1$ equal

$$
(\bar{\pi}, \bar{x})=\left(\pi^{*}+\frac{\lambda}{\kappa}(1-\beta) x^{*}, 0\right) . \text { Thus, }
$$

\footnotetext{
${ }^{23}$ See Eqs. (C.25) and (C.30) and their derivation in Appendix C.
} 


$$
(\bar{\pi}, \bar{x}) \neq\left(\pi^{*}, x^{*}\right) \text { if } x^{*} \neq 0
$$

That is, the social target values, $\pi^{*}$ and $x^{*}$, are uncontrollable, if $x^{*} \neq 0$. The long-run target values, $\bar{\pi}$ and $\bar{x}$, however, prove asymptotically controllable, since $\tilde{\delta}<1$ from Eq. (54).

In sum, when $\phi=1$, time-inconsistency does not exist and we only tackle the issue of controllability. We solve it by delegating the long-run target values and the same weight to the central bank.

Path Controllability with Lagged, Short-Run Target Values When $\phi_{c} \leq \phi \leq 1$. Whether or not the system converges with the long-run target values under the discretionary policy, we consider delegating short-run target values. By Eqs. (21) and (32),

$$
\begin{aligned}
& \pi_{t}^{b}=\bar{\pi}_{t}=\bar{\pi}+\delta\left(\pi_{t-1}-\bar{\pi}\right)=\delta \pi_{t-1}+(1-\delta) \bar{\pi}, \text { and } \\
& x_{t}^{b}=\bar{x}_{t}=\bar{x}+\delta\left(x_{t-1}-\bar{x}\right)=\delta x_{t-1}+(1-\delta) \bar{x} .
\end{aligned}
$$

The short-run target value, $\pi_{t}^{b}\left(x_{t}^{b}\right)$, equals the weighted average of the lagged value, $\pi_{t-1}\left(x_{t-1}\right)$, and the long-run target value, $\bar{\pi}(\bar{x})$. The weight on the lagged value is the system convergence persistence, $\delta$. The corresponding central bank period loss function equals

$$
L_{t}^{b}=\frac{1}{2}\left\{\left[\left(\pi_{t}-\bar{\pi}\right)-\delta\left(\pi_{t-1}-\bar{\pi}\right)\right]^{2}+\lambda^{b}\left[\left(x_{t}-\bar{x}\right)-\delta\left(x_{t-1}-\bar{x}\right)\right]^{2}\right\} .
$$

Because shocks do not affect controllability in the model with a quadratic loss function, a linear the Phillips curve, and additive shocks, we set $u_{t}=0$ and determine the outcomes under a discretionary policy with period loss function (62) as follows ${ }^{24}$

$$
\left(\pi_{t}-\bar{\pi}\right)=\delta\left(\pi_{t-1}-\bar{\pi}\right) \text {, and }
$$

\footnotetext{
${ }^{24}$ See Eqs. (D.10) and (D.12) and their derivation in Appendix D.
} 


$$
\left(x_{t}-\bar{x}\right)=\delta\left(x_{t-1}-\bar{x}\right) .
$$

That is, there exists a discretionary policy, which proves path controllable and, thus, eliminates the constant average and state-contingent inflation biases.

When $u_{t} \neq 0$, the stabilization bias arises. To eliminate biases, using $\tilde{\pi}_{t}\left(u_{t}\right)=\bar{\pi}_{t}\left(u_{t}\right)$ and $\tilde{x}_{t}\left(u_{t}\right)=\bar{x}_{t}\left(u_{t}\right)$ produces $^{25}$

$$
\lambda^{b}=\left(\phi-\phi_{c}\right) \frac{\left(1-\beta \rho^{2}\right)}{\rho(1-\phi \beta \rho)} \lambda,
$$

where $\phi_{c}$ is defined in Eq. $(18 \mathrm{~g})$.

$\lambda^{b}=\lambda$ when $\phi=1$. That is, when $\phi=1$, either the long-run or the short-run target values can achieve path controllability, and the delegated weight always equals the social weight.

Economical feasibility requires $\lambda^{b} \geq 0$. Thus,

$$
\phi \geq \phi_{c} .
$$

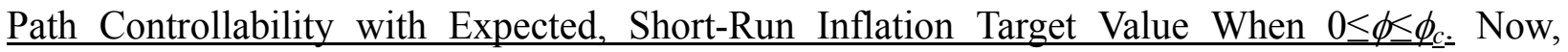
determine the target values, if $\phi \leq \phi_{c}$. Consider more carefully the loss function and the Phillips curve. In the new-classical model with output persistence, the central bank's optimal and controllable loss function involve a term, $\eta x_{t-1}$, which is the important characteristic of the Phillips curve. Accordingly, we guess that the central bank loss function in the principally forward-looking, new-Keynesian model must involve a term, $E_{t} \pi_{t+1}$, which is the main characteristics of the Phillips curve. At the same time, we require path controllability. That is, we require that the target variables evolve at the persistence, $\delta$. As a result, we construct the

\footnotetext{
25 See Eq. (D.13) and its derivation in Appendix D.
} 
short-run target values as follows

$$
\begin{aligned}
& \pi_{t}^{b}=\bar{\pi}+\delta^{-1}\left(E_{t} \pi_{t+1}-\bar{\pi}\right)=\delta^{-1} E_{t} \pi_{t+1}+\left(1-\delta^{-1}\right) \bar{\pi}, \text { and } \\
& x_{t}^{b}=\bar{x}+\delta\left(x_{t-1}-\bar{x}\right)=\delta x_{t-1}+(1-\delta) \bar{x} .
\end{aligned}
$$

The short-run inflation target value equals the 'weighted' $\left(\delta^{-1}>1\right)$ average of the expected inflation rate and the long-run target value. Using an expectation as a target value seems somewhat strange, but we argue that its rationality depends on the forward-looking Phillips curve. Also, the expected inflation target value demonstrates the idea of "implementing optimal policy through inflation-forecast targeting" (Svensson and Woodford, 2005). The corresponding central bank period loss function equals

$$
L_{t}^{b}=\frac{1}{2}\left\{\left[\left(\pi_{t}-\bar{\pi}\right)-\delta^{-1}\left(E_{t} \pi_{t+1}-\bar{\pi}\right)\right]^{2}+\lambda^{b}\left[\left(x_{t}-\bar{x}\right)-\delta\left(x_{t-1}-\bar{x}\right)\right]^{2}\right\} .
$$

Once again, we do not consider shocks and set $u_{t}=0$. The outcomes under a discretionary policy with period loss function (67) equal ${ }^{26}$

$$
\begin{aligned}
& \left(\pi_{t}-\bar{\pi}\right)=\delta\left(\pi_{t-1}-\bar{\pi}\right), \text { and } \\
& \left(x_{t}-\bar{x}\right)=\delta\left(x_{t-1}-\bar{x}\right) .
\end{aligned}
$$

That is, there exists a discretionary policy, which proves path controllable.

When $u_{t} \neq 0$, the stabilization bias arises. To eliminate biases, using $\tilde{\pi}_{t}\left(u_{t}\right)=\bar{\pi}_{t}\left(u_{t}\right)$ and $\tilde{x}_{t}\left(u_{t}\right)=\bar{x}_{t}\left(u_{t}\right)$ produces $^{27}$

$$
\lambda^{b}=\frac{\left(\phi_{c}-\phi\right)\left(1-\beta \rho^{2}\right)}{\delta(1-\delta \beta \rho)(1-\phi \beta \rho)} \lambda .
$$

Economical feasibility requires that $\lambda^{b} \geq 0$. Thus,

\footnotetext{
26 See Eqs. (E.8) and (E.10) and their derivation in Appendix E.

27 See Eq. (E.11) and its derivation in Appendix E.
} 


$$
\phi \leq \phi_{c} .
$$

In sum, the short-run inflation target value conforms to the macroeconomic structure (i.e., Phillips curve). That is, it is lagged (expected), if the inflation rate in the Phillips curve exhibits principally backward-looking (forward-looking) behavior. With the delegated loss function, a discretionary policy proves path controllable (removing the constant average and statecontingent inflation biases), and eliminates stabilization bias, resulting in Ramsey optimality.

The Delegated Weight for $0 \leq \phi \leq 1$. When the Phillips curve exhibits purely backward-looking behavior with no expectations $(\phi=1)$, the time-inconsistency problem does not exist and neither does the stabilization bias. Thus, $\lambda^{b}=\lambda$.

When the Phillips curve exhibits principally backward-looking behavior with an element of expectations ( $\left.\phi_{c} \leq \phi<1\right)$, the time-inconsistency problem and, thus, the stabilization bias exist, and the delegated weight must differ from the social weight $\left(\lambda^{b} \neq \lambda\right)$. The coefficient of the weight $\lambda^{b}$ depends only on the dynamic parameters $(\phi, \beta$, and $\rho$ ), and does not relate to the system convergence persistence, $\delta$. By Eq. (64),

$$
\lambda^{b}=\frac{\left(\phi-\phi_{c}\right)\left(1-\beta \rho^{2}\right)}{\rho(1-\phi \beta \rho)} \lambda=\left[1-\frac{(1-\phi)}{\rho(1-\phi \beta \rho)}\right] \lambda<\lambda .
$$

Therefore, the central bank must exhibit conservatism $\left(\lambda^{b}<\lambda\right)$. Intuitively, if the inflation rate persists $\left(\phi_{c} \leq \phi<1\right)$, then current inflation rate deviation from its target value will persist into future and, thus, cause losses. To reduce these losses, we must place more weight on the inflation rate target (i.e., $\lambda^{b}<\lambda$ ). In the new-classical model with output persistence, we must place more weight on the output gap because of output gap persistence. 
When the Phillips curve exhibits principally forward-looking behavior $\left(0<\phi \leq \phi_{c}\right)$, the weight in Eq. (69) depends not only on the dynamic parameters but also on the system convergence persistence. The weight becomes subtle because of forward-looking behavior. No analytical way exists to discuss the relationship of the weight with model parameters.

For the purely forward-looking case $(\phi=0)$, the central bank exhibit weight-conservative (weight-liberal) behavior, if the cost-push shock more (less) persists. The delegated weight in Eq. (69) reduces to

$$
\lambda^{b}=\frac{(1-\rho)}{\delta(1-\delta \beta \rho)} \lambda .
$$

We report the following conditions

$$
\begin{aligned}
& \lambda^{b}<\lambda \quad \text { if } \rho>\rho_{c}, \\
& \lambda^{b}=\lambda \quad \text { if } \rho=\rho_{c}, \text { and } \\
& \lambda^{b}>\lambda \quad \text { if } \rho<\rho_{c}, \text { where } \\
& \rho_{c}=\frac{1-\delta}{1-\beta \delta^{2}}, \text { and } 0<\rho_{c}<1 .
\end{aligned}
$$

The critical value, $\rho_{c}$, depends on model parameters. The central bank is weightconservative (weight-liberal), if cost-push shock persistence is greater (less) than the critical value. Actually, $\partial \lambda^{b} / \partial \rho<0{ }^{28}$ A more persistent cost-push shock implies less weight on output stabilization. To see this, iterate the Phillips curve (Eq. 13) with $\phi=0$ forward as follows

$$
\pi_{t}=E_{t} \sum_{i=0}^{\infty} \beta^{i}\left(\kappa x_{t+i}+u_{t+i}\right) .
$$

${ }^{28} \partial \lambda^{b} / \partial \rho=-\lambda(1-\beta \delta) /\left[\delta(1-\delta \beta \rho)^{2}\right]<0$ 
Inflation depends entirely on current and expected future output gaps and cost-push shocks, because of the purely forward-looking nature of the Phillips curve. Thus, more persistent cost-push shocks imply that more losses occur because more inflation increases emerge, driven by current and future cost-push shocks. To reduce the losses caused by current and future inflation, the policy makers must place more weight on inflation stabilization and, thus, less weight on output stabilization.

\section{Situations of the Same Weight $\left(\lambda^{b}=\lambda\right)$}

A summary of the situations of the same weight $\left(\lambda^{b}=\lambda\right)$ provides more insight about delegation. We determine two situations where $\lambda^{b}=\lambda$-- static models in a game context and models in a one-decision-maker context.

\section{Static Models in a Game Context}

Consider the delegated weight, $\lambda^{b}=\lambda /\left(1-\beta \eta^{2}\right)$, in the new-classical model with output persistence. Obviously, $\lambda^{b}=\lambda$, when $\beta=0$ and/or $\eta=0$.

When the central bank does not care about future losses $(\beta=0)$, the model, which includes the loss function, the Phillips curve, and rational expectations, is essentially static even if the Phillips curve exhibits output persistence $(\eta \neq 0)$. When persistence does not enter the Phillips curve $(\eta=0)$, the model is also essentially static even if the central bank cares about the future losses $(\beta \neq 0)$. In a word, the model is static when $\beta=0$ and/or $\eta=0$. The time-inconsistency problem, however, always exists because of the assumption of rational expectations.

We know that the stabilization bias arises, which requires a different weight to eliminate it, when the time-inconsistency problem exists. Why does the delegated weight equal the social 
one? We argue that discretionary policy need not trade off over time with a static model and need not alter the balance between inflation and output-gap stabilization when we delegate the correct short-run target values $\left(\pi_{t}^{b}=\pi^{*}\right.$ and $\left.x_{t}^{b}=\eta x_{t-1}\right)$. As a result, $\lambda^{b}=\lambda$.

The correct target values are important as the literature stresses. An overly ambitious output (employment) target value produces inflation bias. Rogoff's (1985) weight-conservative approach tackles this overly ambitious employment target value by placing less (more) weight on the employment (inflation) stabilization. Rather than adjusting the weight, we tackle the problem directly by correcting the overly ambitious employment target value. With correct target values, the central bank does not need a different weight to balance two target variables' stabilization in a static model.

The two static game models in Yuan, et al. (2011), where the time-inconsistency problem exists, also demonstrate that the delegated weight equal the social weight when we delegate the central bank the correct target values.

\section{Models in a One-Decision-Maker Context}

Consider the hybrid new-Keynesian model with $\phi=1$. In this situation, $\lambda^{b}=\lambda$. When $\phi=1$, the Phillips curve contains no expectations and, thus, the time-inconsistency problem and the stabilization bias do not exist. As a result, the central bank adopts the same weight as society.

As an exercise, we consider the hybrid new-Keynesian model with $\beta=0$. When $\beta=0$, the Phillips curve contains no expectations and, thus, the time-inconsistency problem does not exist. 
Thus, $\lambda^{b}=\lambda$ in this situation. ${ }^{29}$

Based on the two situations of $\lambda^{b}=\lambda$-- static models in a game context and models with one decision maker, we conclude that $\lambda^{b} \neq \lambda$ in a dynamic game model. Moreover, the coefficient of the delegated weight depends on dynamic parameters and/or the system convergence persistence.

\section{Conclusion}

In this paper, we tackle two issues in policymaking - time-inconsistency and target controllability - in two dynamic models. Time-inconsistency results from expectations (multiple decision makers). Target controllability arises because the target variables must conform to the constraint imposed by the economic model (the Phillips curve). We can resolve both issues by delegating a loss function, which differs from the social loss function, to the central bank. The delegation scheme is simple with one decision maker whereas it is relatively complicated in a game context.

We obtain propositions about target controllability. That is, if the system, which consists of a quadratic loss function and a linear Phillips curve, converges under the Ramsey policy or under discretionary policy, constant target values are jointly asymptotically controllable if and only if they satisfy the Phillips curve in equilibrium. We find that the target variables' equilibriums under the Ramsey policy satisfy the Phillips curve in equilibrium and, thus, are jointly asymptotically controllable. We call these equilibrium values the long-run target values.

With one decision maker as in the hybrid new-Keynesian model with $\phi=1$, the model

29 See Eq. (F.9) in Appendix F for the derivation. 
involves no expectations and exhibits purely backward-looking behavior. In this context, the time-inconsistency problem does not exist, and we only need to tackle the target controllability problem. Delegating the long-run target values (the target variables' equilibriums under the Ramsey policy) to the central bank can produce not only asymptotic but also path controllability. Delegating short-run target values proves unnecessary because time-inconsistency does not exist. Moreover, the central bank adopts the same weight as society because stabilization bias does not exist.

With multiple decision makers where the model involves expectations and, thus, timeinconsistency exists, the long-run target values can only achieve asymptotic rather than path controllability. As a result, we delegate short-run, state-contingent target values to the central bank to obtain path controllability. They commit and bind the central bank to follow exactly the Ramsey optimal paths. The short-run inflation target value conforms to the macroeconomic structure (i.e., Phillips curve). That is, it is lagged (or expected, or exactly the social target value) if the inflation rate exhibits principally backward-looking (or forward-looking, or rational expectations) behavior in the Phillips curve. ${ }^{30}$ Specifically, the short-run inflation target value equals the weighted (or 'weighted' in form) average of the lagged (or the expected) value and its long-run target value, if the inflation rate exhibits principally backward-looking (or forwardlooking) behavior in the Phillips curve as in the new-Keynesian model with $\phi_{c} \leq \phi<1$ (or $0 \leq \phi \leq \phi_{c}$ ). The weight on the lagged value equals the system convergence persistence under the

\footnotetext{
${ }^{30}$ In a model (Phillips curve) with principally forward-looking behavior, the delegated inflation target value is expected, demonstrating the idea of "implementing optimal policy through inflation-forecast targeting" (Svensson and Woodford, 2005).
} 
Ramsey policy whereas the 'weight' on the expected value equals the reciprocal of the persistence. The inflation target value always equals the social target value, if the private sector forms rationally expectations about the inflation as in the new-classical model. With regards to the output gap, the short-run target value equals the weighted average of the lagged value and its long-run target value. In a word, the short-run target values converge to the long-run target values and, though state-contingent, are predetermined. With the short-run target values, the discretionary policy proves path controllable and, thus, eliminate the constant average and state-contingent inflation biases.

Delegating a different weight to the central bank can eliminate stabilization bias in a dynamic game model. When the output gap exhibits persistent, the central bank must place more weight on the output gap stabilization as in the new-classical model. When the inflation rate exhibits more persistence than expectation, the central bank must place more weight on inflation rate stabilization as in the hybrid new-Keynesian model with $\phi_{c} \leq \phi<1$. The intuition is straightforward. When the output gap (inflation rate) exhibits persistent, current deviation from its target value will persist into future, and induce losses. To reduce the losses, the central bank must place more weight on output-gap (inflation) stabilization. Moreover, the coefficients of the weights in these two situations depend only on the dynamic parameters, and do not relate to the system convergence persistence.

When the inflation rate exhibits principally forward-looking behavior as in the hybrid new-Keynesian model with $0 \leq \phi \leq \phi_{c}$, the delegated weight becomes subtle and the central bank may adopt a liberal or conservative weight, depending on both the dynamic parameters and 
the system convergence persistence.

We determine two situations where the delegated weight equals the social weight $\left(\lambda^{b}=\lambda\right)$ -- static models in a game context and models with one decision maker. As a result, we conclude that the delegated weight must differ from society $\left(\lambda^{b} \neq \lambda\right)$ in a dynamic game model. The coefficient of the delegated weight depends on dynamic parameters and/or the system convergence persistence.

In sum, we can solve both issues - time-inconsistency and target controllability - by delegation. Through delegation, discretionary policy proves path controllable (removing the constant average and state-contingent inflation biases) and eliminates stabilization bias, resulting in Ramsey optimality.

\section{Appendix A: Ramsey Optimal Solution in a Hybrid New-Keynesian Model}

The optimization problem minimizes the social intertemporal loss function (12) with period loss function (2) subject to the Phillips curve (Eq. 13). Its Lagrangian expression equals the following

$$
\mathfrak{L}=E_{0} \sum_{t=0}^{\infty} \beta^{t}\left\{\begin{array}{l}
\frac{1}{2}\left[\left(\pi_{t}-\pi^{*}\right)^{2}+\lambda\left(x_{t}-x^{*}\right)^{2}\right] \\
+\psi_{t}\left[\kappa x_{t}+\phi \pi_{t-1}+(1-\phi) \beta \pi_{t+1}+u_{t}-\pi_{t}\right]
\end{array}\right\} .
$$

The first-order conditions equal

$$
\frac{\partial \mathfrak{L}}{\partial x_{t}}=E_{0}\left\{\beta^{t}\left[\lambda\left(x_{t}-x^{*}\right)+\kappa \psi_{t}\right]\right\}=0 \quad \text { for } t \geq 0
$$

$$
\begin{array}{ll}
\frac{\partial \mathfrak{L}}{\partial \pi_{0}}=E_{0}\left[\left(\pi_{0}-\pi^{*}\right)-\psi_{0}+\beta \phi \psi_{1}\right]=0 & \text { for } t=0, \text { and } \\
\frac{\partial \mathfrak{L}}{\partial \pi_{t}}=E_{0}\left\{\begin{array}{ll}
\beta^{t}\left[\left(\pi_{t}-\pi^{*}\right)-\psi_{t}\right] \\
+\beta^{t+1} \phi \psi_{t+1}+\beta^{t-1}(1-\phi) \beta \psi_{t-1}
\end{array}\right\}=0 & \text { for } t \geq 1 .
\end{array}
$$


Eliminating the multipliers from Eqs. (A.2) gives the consolidated first-order conditions as follows

$$
\begin{array}{ll}
\left(x_{0}-x^{*}\right)-\phi \beta\left(E_{0} x_{1}-x^{*}\right)=-\frac{\kappa}{\lambda}\left(\pi_{0}-\pi^{*}\right) & \text { for } t=0, \text { and } \\
\left(E_{0} x_{t}-x^{*}\right)-\phi \beta\left(E_{0} x_{t+1}-x^{*}\right)-(1-\phi)\left(E_{0} x_{t-1}-x^{*}\right)=-\frac{\kappa}{\lambda}\left(E_{0} \pi_{t}-\pi^{*}\right) & \text { for } t \geq 1 .
\end{array}
$$

Combining Eqs. (A.3a) and (A.3b) with the Phillips curve (Eq. 13) produces

(A.4a)

$$
\begin{aligned}
& a \beta^{2} E_{0} x_{2}-\beta E_{0} x_{1}+\left[1+\frac{\kappa^{2}}{\lambda}+(1-\phi)^{2} \beta\right] x_{0}+\frac{\kappa}{\lambda} u_{0}-\phi \frac{\kappa}{\lambda}\left(\pi^{*}-\pi_{-1}\right) \\
& -(1-\phi)(1-\beta)\left[\frac{\kappa}{\lambda} \pi^{*}+\phi(1-\beta) x^{*}\right]-\left[(1-\phi)+\phi^{2}(1-\beta)\right] x^{*}=0 \text { for } t=0 \text {, and } \\
& a \beta^{2} E_{0} x_{t+2}-\beta E_{0} x_{t+1}+b E_{0} x_{t}-E_{0} x_{t-1}+a E_{0} x_{t-2} \\
& -(1-\phi)(1-\beta)\left[\frac{\kappa}{\lambda} \pi^{*}+\phi(1-\beta) x^{*}\right]+\frac{\kappa}{\lambda} E_{0} u_{t}=0 \quad \text { for } t \geq 1 \text {, where }
\end{aligned}
$$

$$
a \equiv \phi(1-\phi)
$$

$$
b \equiv 1+\frac{\kappa^{2}}{\lambda}+\phi^{2} \beta+(1-\phi)^{2} \beta=1+\beta+\frac{\kappa^{2}}{\lambda}-2 a \beta, \text { and }
$$

$$
X_{-1} \equiv 0 \text {. }
$$

Solve Eqs. (A.4a) and (A.4b) backwards. Assume that $\delta$ equals a root of the characteristic equation

$$
a \beta^{2} \delta^{4}-\beta \delta^{3}+b \delta^{2}-\delta+a=0 .
$$

Assume that the solution of Eq. (A.4b) for $t \geq 1$ takes the following form:

$$
x_{t}=\delta x_{t-1}+e+f u_{t} .
$$

Using Eq. (A.7) and $E_{0} u_{t}=\rho E_{0} u_{t-1}$ leads to

$$
E_{0} x_{t}=\delta E_{0} x_{t-1}+e+f \rho E_{0} u_{t-1},
$$


(A.8b)

$$
E_{0} X_{t+1}=\delta^{2} E_{0} X_{t-1}+(\delta+1) e+(\delta+\rho) f \rho E_{0} u_{t-1} \text {, and }
$$

$$
E_{0} X_{t+2}=\delta^{3} E_{0} X_{t-1}+\left(\delta^{2}+\delta+1\right) e+\left(\delta^{2}+\delta \rho+\rho^{2}\right) f \rho E_{0} u_{t-1} .
$$

Substituting Eqs. (A.8a), (A.8b), (A.8c), and $E_{0} u_{t}=\rho E_{0} u_{t-1}$ into Eq. (A.4b) results in

$$
\begin{aligned}
\left(a \beta^{2} \delta^{3}-\beta \delta^{2}\right. & +b \delta-1) E_{0} x_{t-1}+a E_{0} x_{t-2}+\left(d f+\frac{\kappa}{\lambda}\right) \rho E_{0} u_{t-1} \\
& +c e-(1-\phi)(1-\beta)\left[\frac{\kappa}{\lambda} \pi^{*}+\phi(1-\beta) x^{*}\right]=0, \text { where }
\end{aligned}
$$

$$
\begin{aligned}
& c \equiv a \beta^{2}\left(\delta^{2}+\delta+1\right)-\beta(\delta+1)+b, \text { and } \\
& d \equiv a \beta^{2}\left(\delta^{2}+\delta \rho+\rho^{2}\right)-\beta(\delta+\rho)+b .
\end{aligned}
$$

Transforming Eq. (A.9) and noting that $-1 /\left(a \beta^{2} \delta^{3}-\beta \delta^{2}+b \delta-1\right)=\delta / a$ from Eq.

(A.6) produces

$$
\begin{aligned}
E_{0} x_{t-1}=\delta E_{0} x_{t-2}+ & \frac{\delta}{a}\left\{c e-(1-\phi)(1-\beta)\left[\frac{\kappa}{\lambda} \pi^{*}+\phi(1-\beta) x^{*}\right]\right\} \\
+ & \frac{\delta}{a}\left(d f+\frac{\kappa}{\lambda}\right) \rho E_{0} u_{t-1} .
\end{aligned}
$$

Comparing Eq. (A.11) with Eq. (A.7) implies

$$
e=\frac{\delta(1-\phi)(1-\beta)}{\delta c-a}\left[\frac{\kappa}{\lambda} \pi^{*}+\phi(1-\beta) x^{*}\right] \text {, and }
$$

$$
f=-\frac{\kappa \delta \rho}{\lambda(\delta \rho d-a)}
$$

To interpret the constant $e$ in Eq. (A.7), we compute

$$
\bar{x} \equiv \frac{e}{1-\delta}=\frac{\delta(1-\phi)(1-\beta)}{(\delta c-a)(1-\delta)}\left[\frac{\kappa}{\lambda} \pi^{*}+\phi(1-\beta) x^{*}\right] .
$$

With the notation $\bar{x}$, the solution of the output gap for $t \geq 1$ equals

$$
\left(x_{t}-\bar{x}\right)=\delta\left(x_{t-1}-\bar{x}\right)+f u_{t},
$$


where $\delta, \bar{x}$ and $f$ are defined respectively in Eqs. (A.6), (A.13 or A.19) and (A.12b).

To obtain another form of $\bar{x}$, compute

$$
\begin{aligned}
\delta c-a & =\delta\left[a \beta^{2}\left(\delta^{2}+\delta+1\right)-\beta(\delta+1)+b\right]-a \\
& =\left(a \beta^{2} \delta^{3}-\beta \delta^{2}+b \delta\right)+a \beta^{2} \delta^{2}+a \beta^{2} \delta-\beta \delta-a, \text { and }
\end{aligned}
$$

Noting that $a \beta^{2} \delta^{4}-\beta \delta^{3}+b \delta^{2}=\delta-a$ from Eq. (A.6), (A.15) becomes

$$
\begin{aligned}
(\delta c-a) \delta & =\left(a \beta^{2} \delta^{4}-\beta \delta^{3}+b \delta^{2}\right)+a \beta^{2} \delta^{3}+a \beta^{2} \delta^{2}-\beta \delta^{2}-a \delta \\
& =\delta-a+a \beta^{2} \delta^{3}+a \beta^{2} \delta^{2}-\beta \delta^{2}-a \delta .
\end{aligned}
$$

Subtracting Eq. (A.16) from Eq. (A.15) gives

$$
(\delta c-a)(1-\delta)=\left(a \beta^{2}+b-\beta-1+a\right) \delta .
$$

Substituting $a \beta^{2}+b-\beta-1+a=\frac{\kappa^{2}}{\lambda}+a(1-\beta)^{2}$ from Eq. (A.5b) into Eq. (A.17) yields

$$
(\delta c-a)(1-\delta)=\left[\frac{\kappa^{2}}{\lambda}+a(1-\beta)^{2}\right] \delta .
$$

Substituting Eq. (A.18) into Eq. (A.13) gives

$$
\bar{x} \equiv \frac{e}{1-\delta}=(1-\phi)(1-\beta)\left[\frac{\kappa \pi^{*}+\lambda \phi(1-\beta) x^{*}}{\kappa^{2}+\lambda a(1-\beta)^{2}}\right] .
$$

Solve for $x_{0}$. Applying expectations $E_{0}$ to Eq. (A.7) for $t=1$ and $t=2$ generates

$$
\begin{aligned}
& E_{0} x_{1}=\delta x_{0}+e+f \rho u_{0}=\delta x_{0}+(1-\delta) \bar{x}+f \rho u_{0}, \text { and } \\
& E_{0} x_{2}=\delta E_{0} x_{1}+e+f \rho^{2} u_{0}=\delta^{2} x_{0}+\left(1-\delta^{2}\right) \bar{x}+(\delta+\rho) f \rho u_{0} .
\end{aligned}
$$

Substituting $(1-\phi)(1-\beta)\left[\frac{\kappa}{\lambda} \pi^{*}+\phi(1-\beta) x^{*}\right]=\left[\frac{\kappa^{2}}{\lambda}+a(1-\beta)^{2}\right] \bar{x}$ from Eq. (A.19),

Eqs. (A.20a) and (A.20b) into Eq. (A.4a) for $t=0$ leads to 


$$
\begin{aligned}
& \left\{a \beta^{2} \delta^{2}-\beta \delta+\left[1+\frac{\kappa^{2}}{\lambda}+(1-\phi)^{2} \beta\right]\right\} x_{0} \\
& -\left[a \beta^{2} \delta^{2}-\beta \delta+a+\left(\beta+\frac{\kappa^{2}}{\lambda}-2 a \beta\right)\right] \bar{x}-\phi \frac{\kappa}{\lambda}\left(\pi^{*}-\pi_{-1}\right) \\
& -\left(1-a-\phi^{2} \beta\right) x^{*}+a \beta^{2}(\delta+\rho) f \rho u_{0}-f \beta \rho u_{0}+\frac{\kappa}{\lambda} u_{0}=0 .
\end{aligned}
$$

Substituting $1+\frac{\kappa^{2}}{\lambda}+(1-\phi)^{2} \beta=b-\phi^{2} \beta$ and $\beta+\frac{\kappa^{2}}{\lambda}-2 a \beta=b-1$ from Eq. (A.5b), $\frac{\kappa}{\lambda}=-f\left(d-\frac{a}{\delta \rho}\right)$ from Eq. (A.12b) and $d$ in Eq. (A.10b) into Eq. (A.21) generates

$$
\begin{aligned}
\left(a \beta^{2} \delta^{2}-\beta \delta\right. & \left.+b-\phi^{2} \beta\right)\left(x_{0}-\bar{x}\right)=\left(1-a-\phi^{2} \beta\right)\left(x^{*}-\bar{x}\right) \\
+ & \phi \frac{\kappa}{\lambda}\left(\pi^{*}-\pi_{-1}\right)+\left(a \beta^{2} \delta^{2}-\beta \delta+b-\frac{a}{\delta \rho}\right) f u_{0} .
\end{aligned}
$$

Denote

(A.23a)

$$
\begin{aligned}
\delta_{x 0} & =\frac{\left(1-a-\phi^{2} \beta\right)}{\left(a \beta^{2} \delta^{2}-\beta \delta+b-\phi^{2} \beta\right)}, \text { and } \\
f_{0} \equiv & \frac{\left(a \beta^{2} \delta^{2}-\beta \delta+b-\frac{a}{\delta \rho}\right)}{\left(a \beta^{2} \delta^{2}-\beta \delta+b-\phi^{2} \beta\right)} f=-\frac{\kappa\left[\left(a \beta^{2} \delta^{2}-\beta \delta+b\right) \delta \rho-a\right]}{\lambda\left(a \beta^{2} \delta^{2}-\beta \delta+b-\phi^{2} \beta\right)(\delta \rho d-a)} .
\end{aligned}
$$

With the notations, Eq. (A.22) becomes

$$
\left(x_{0}-\bar{x}\right)=\delta_{x 0}\left[\left(x^{*}-\bar{x}\right)+\frac{\phi \kappa\left(\pi^{*}-\pi_{-1}\right)}{\lambda\left(1-a-\phi^{2} \beta\right)}\right]+f_{0} u_{0} .
$$

Note that $\delta_{x 0}=\delta$ and Eq. (A.24) reduces to $\left(x_{0}-\bar{x}\right)=\delta\left(x^{*}-\bar{x}\right)+f u_{0}$, when $\phi=0$.

Now, solve for $\pi_{t}$. Combining Eqs. (A.3a) and (A.3b) with the Phillips curve (Eq. 13) and using $E_{0} u_{t+1}=\rho E_{0} u_{t}$ generates 
(A.25a)

$$
\begin{aligned}
& a \beta^{2} E_{0} \pi_{2}-\beta E_{0} \pi_{1}+\left(1+\frac{\kappa^{2}}{\lambda}+\phi^{2} \beta\right) \pi_{0}-(1-\phi \beta \rho) u_{0} \\
& -\kappa\left[\frac{\kappa}{\lambda} \pi^{*}+\phi(1-\beta) x^{*}\right]-\phi \pi_{-1}-(1-\phi) \kappa x^{*}=0 \quad \text { for } t=0, \text { and } \\
& a \beta^{2} E_{0} \pi_{t+2}-\beta E_{0} \pi_{t+1}+b E_{0} \pi_{t}-E_{0} \pi_{t-1}+a E_{0} \pi_{t-2} \\
& -\kappa\left[\frac{\kappa}{\lambda} \pi^{*}+\phi(1-\beta) x^{*}\right]-(1-\phi \beta \rho) E_{0} u_{t}+(1-\phi) E_{0} u_{t-1}=0 \text { for } t \geq 1,
\end{aligned}
$$

where $a$ and $b$ are defined, respectively, in Eqs. (A.5a) and (A.5b).

Solve Eqs. (A.25a) and (A.25b) backwards. The characteristic equation of Eq. (A.25b) equals Eq. (A.6). Assume that the solution of Eq. (A.25b) takes the following form

$$
\pi_{t}=\delta \pi_{t-1}+g+h\left[(1-\phi \beta \rho) u_{t}-(1-\phi) u_{t-1}\right],
$$

where $\delta$ is a root of the characteristic equation (A.6).

Using Eq. (A.26) and $E_{0} u_{t}=\rho E_{0} u_{t-1}$ leads to

$$
\begin{aligned}
& E_{0} \pi_{t}=\delta E_{0} \pi_{t-1}+g+h \rho\left[(1-\phi \beta \rho) E_{0} u_{t-1}-(1-\phi) E_{0} u_{t-2}\right], \\
& E_{0} \pi_{t+1}=\delta^{2} E_{0} \pi_{t-1}+(\delta+1) g+(\delta+\rho) h \rho\left[(1-\phi \beta \rho) E_{0} u_{t-1}-(1-\phi) E_{0} u_{t-2}\right], \text { and } \\
& E_{0} \pi_{t+2}=\delta^{3} E_{0} \pi_{t-1}+\left(\delta^{2}+\delta+1\right) g \\
&+\left(\delta^{2}+\delta \rho+\rho^{2}\right) h \rho\left[(1-\phi \beta \rho) E_{0} u_{t-1}-(1-\phi) E_{0} u_{t-2}\right] .
\end{aligned}
$$

Substituting Eqs. (A.27a) to (A.27c), and $E_{0} u_{t}=\rho E_{0} u_{t-1}$ into Eq. (A.25b) results in

$$
\begin{gathered}
\left(a \beta^{2} \delta^{3}-\beta \delta^{2}+b \delta-1\right) E_{0} \pi_{t-1}+a E_{0} \pi_{t-2}+c g-\kappa\left[\frac{\kappa}{\lambda} \pi^{*}+\phi(1-\beta) x^{*}\right] \\
+(d h-1) \rho\left[(1-\phi \beta \rho) E_{0} u_{t-1}-(1-\phi) E_{0} u_{t-2}\right]=0
\end{gathered}
$$

where $c$ and $d$ are defined respectively in Eqs. (A.10a) and (A.10b).

Transforming Eq. (A.28) and noting that $-1 /\left(a \beta^{2} \delta^{3}-\beta \delta^{2}+b \delta-1\right)=\delta / a$ from Eq.

(A.6) produces 
(A.29)

$$
\begin{aligned}
E_{0} \pi_{t-1}=\delta E_{0} \pi_{t-2} & +\frac{\delta}{a}\left\{c g-\kappa\left[\frac{\kappa}{\lambda} \pi^{*}+\phi(1-\beta) x^{*}\right]\right\} \\
+ & \frac{\delta}{a}(d h-1) \rho\left[(1-\phi \beta \rho) E_{0} u_{t-1}-(1-\phi) E_{0} u_{t-2}\right] .
\end{aligned}
$$

Comparing Eq. (A.29) with Eq. (A.26) implies

$$
g=\frac{\kappa \delta}{\delta c-a}\left[\frac{\kappa}{\lambda} \pi^{*}+\phi(1-\beta) x^{*}\right] \text {, and }
$$

$$
h=\frac{\delta \rho}{\delta \rho d-a} .
$$

Substituting $\frac{\delta}{(\delta c-a)}=\frac{\lambda(1-\delta)}{\kappa^{2}+\lambda a(1-\beta)^{2}}$ from Eq. (A.18) into Eq. (A.30a) produces

$$
g=\kappa(1-\delta)\left[\frac{\kappa \pi^{*}+\lambda \phi(1-\beta) x^{*}}{\kappa^{2}+\lambda a(1-\beta)^{2}}\right] .
$$

Denote

$$
\bar{\pi} \equiv \frac{g}{1-\delta}=\kappa\left[\frac{\kappa \pi^{*}+\lambda \phi(1-\beta) x^{*}}{\kappa^{2}+\lambda a(1-\beta)^{2}}\right] .
$$

Using the AR(1) process of shocks in Eq. (14), the term that involves shocks in Eq. (A.26)

produces

$$
\begin{aligned}
& (1-\phi \beta \rho) u_{t}-(1-\phi) u_{t-1}=\left(\phi-\phi_{c}\right)\left(1-\beta \rho^{2}\right) u_{t-1}+(1-\phi \beta \rho) \hat{u}_{t}, \text { where } \\
& \phi_{c}=\frac{1-\rho}{1-\beta \rho^{2}}, \text { and } 0<\phi_{c} \leq 1 .
\end{aligned}
$$

With the notation $\bar{\pi}$, the solution of the inflation in Eq. (A.26) for $t \geq 1$ equals

$$
\begin{aligned}
& \left(\pi_{t}-\bar{\pi}\right)=\delta\left(\pi_{t-1}-\bar{\pi}\right)+h\left[(1-\phi \beta \rho) u_{t}-(1-\phi) u_{t-1}\right], \quad \text { or } \\
& \left(\pi_{t}-\bar{\pi}\right)=\delta\left(\pi_{t-1}-\bar{\pi}\right)+h\left[\left(\phi-\phi_{c}\right)\left(1-\beta \rho^{2}\right) u_{t-1}+(1-\phi \beta \rho) \hat{u}_{t}\right],
\end{aligned}
$$

where $\delta, \bar{\pi}, \phi_{c}$, and $h$ are defined respectively in Eqs. (A.6), (A.32), (A.34) and (A.30b).

Solve for $\pi_{0}$. Applying expectations $E_{0}$ to Eq. (A.35) for $t=1$ and $t=2$ generates 
(A.36a)

$$
E_{0} \pi_{1}=\delta \pi_{0}+(1-\delta) \bar{\pi}+h\left(\phi-\phi_{c}\right)\left(1-\beta \rho^{2}\right) u_{0} \text {, and }
$$

$$
E_{0} \pi_{2}=\delta^{2} \pi_{0}+\left(1-\delta^{2}\right) \bar{\pi}+(\delta+\rho) h\left(\phi-\phi_{c}\right)\left(1-\beta \rho^{2}\right) u_{0} .
$$

Substituting $\kappa\left[\frac{\kappa}{\lambda} \pi^{*}+\phi(1-\beta) x^{*}\right]=\left[\frac{\kappa^{2}}{\lambda}+a(1-\beta)^{2}\right] \bar{\pi}$ from Eq. (A.32), Eqs. (A.36a) and (A.36b) into Eq. (A.25a) for $t=0$ and arranging leads to

$$
\begin{aligned}
& {\left[a \beta^{2} \delta^{2}-\beta \delta+\left(1+\frac{\kappa^{2}}{\lambda}+\phi^{2} \beta\right)\right] \pi_{0}-\left[a \beta^{2} \delta^{2}-\beta \delta+a+\left(\beta+\frac{\kappa^{2}}{\lambda}-2 a \beta\right)\right] \bar{\pi}} \\
& -\phi \pi_{-1}-(1-\phi) \kappa x^{*}-\left\{\beta[1-a \beta(\delta+\rho)] h\left(\phi-\phi_{c}\right)\left(1-\beta \rho^{2}\right)+(1-\phi \beta \rho)\right\} u_{0}=0 .
\end{aligned}
$$

Substituting $1+\frac{\kappa^{2}}{\lambda}+\phi^{2} \beta=b-(1-\phi)^{2} \beta$ and $\beta+\frac{\kappa^{2}}{\lambda}-2 a \beta=b-1$ from Eq. (A.5b) into Eq. (A.37) yields

$$
\begin{aligned}
& {\left[a \beta^{2} \delta^{2}-\beta \delta+b-(1-\phi)^{2} \beta\right]\left(\pi_{0}-\bar{\pi}\right)+(1-a) \bar{\pi}-(1-\phi)(1-\phi) \beta \bar{\pi}} \\
& -\phi \pi_{-1}-(1-\phi) \kappa x^{*}-\left\{\beta[1-a \beta(\delta+\rho)] h\left(\phi-\phi_{c}\right)\left(1-\beta \rho^{2}\right)+(1-\phi \beta \rho)\right\} u_{0}=0 .
\end{aligned}
$$

From Eqs. (A.19) and (A.32), $\kappa \bar{x}=(1-\phi)(1-\beta) \bar{\pi}$. Thus, $(1-\phi) \beta \bar{\pi}=(1-\phi) \bar{\pi}-\kappa \bar{x}$.

Substituting it into Eq. (A.38) produces

$$
\begin{aligned}
& \left(\pi_{0}-\bar{\pi}\right)=\delta_{\pi 0}\left[\phi\left(\pi_{-1}-\bar{\pi}\right)+(1-\phi) \kappa\left(x^{*}-\bar{x}\right)\right]+h_{0} u_{0}, \text { where } \\
& \delta_{\pi 0} \equiv \frac{1}{a \beta^{2} \delta^{2}-\beta \delta+b-(1-\phi)^{2} \beta}, \text { and } \\
& h_{0} \equiv \frac{\beta[1-a \beta(\delta+\rho)] h\left(\phi-\phi_{c}\right)\left(1-\beta \rho^{2}\right)+(1-\phi \beta \rho)}{a \beta^{2} \delta^{2}-\beta \delta+b-(1-\phi)^{2} \beta} .
\end{aligned}
$$

Note that $\delta_{x 0}=\delta$ and Eq. (A.39) reduces to $\left(\pi_{0}-\bar{\pi}\right)=\delta\left(\pi_{-1}-\bar{\pi}\right)+h_{0} u_{0}$, when $\phi=1$. 


\section{Appendix B: Discretionary Policy with State-Contingent, Short-Run Target Values}

\section{in the New-Classical Model with Output Persistence}

The following specifies the problem of the central bank with discretion

$$
\begin{aligned}
& \min _{\left\{\pi_{t}\right\}_{t=1}^{\infty}} E_{0}\left\{\sum_{t=1}^{\infty} \beta^{t-1} \frac{1}{2}\left[\left(\pi_{t}-\pi^{*}\right)^{2}+\lambda^{b}\left(x_{t}-\eta x_{t-1}\right)^{2}\right]\right\} \\
& \text { s.t. }\left\{\begin{array}{l}
x_{t}=\eta x_{t-1}+\alpha\left(\pi_{t}-\pi_{t}^{e}\right)+u_{t}, \\
\pi_{t}^{e}=E_{t-1} \pi_{t} .
\end{array}\right.
\end{aligned}
$$

The Bellman equation for determining the discretionary policy equals

$$
\begin{aligned}
& V\left(x_{t-1}\right)=E_{t-1} \min _{\pi_{t}}\left\{\frac{1}{2}\left[\left(\pi_{t}-\pi^{*}\right)^{2}+\lambda^{b}\left(x_{t}-\eta x_{t-1}\right)^{2}\right]+\beta V\left(x_{t}\right)\right\} \text { with } \\
& V\left(x_{t}\right)=\gamma_{0}+\gamma_{1} x_{t}+\frac{1}{2} \gamma_{2} x_{t}^{2} .
\end{aligned}
$$

Its first-order condition becomes

$$
\pi_{t}-\pi^{*}+\lambda^{b} \alpha\left(x_{t}-\eta x_{t-1}\right)+\beta \alpha\left(\gamma_{1}+\gamma_{2} x_{t}\right)=0 \text {. }
$$

Substituting the Phillips curve (Eq. 3) into Eq. (B.4) produces

$$
\pi_{t}-\pi^{*}+\alpha \beta \gamma_{1}+\beta \gamma_{2} \alpha \eta x_{t-1}+\alpha^{2}\left(\lambda^{b}+\beta \gamma_{2}\right)\left(\pi_{t}-\pi_{t}^{e}\right)+\alpha\left(\lambda^{b}+\beta \gamma_{2}\right) u_{t}=0 .
$$

Taking expectations $E_{t-1}$ of Eq. (B.5) gives

$$
\pi_{t}^{e}=\pi^{*}-\alpha \beta \gamma_{1}-\beta \gamma_{2} \alpha \eta x_{t-1}
$$

Substituting Eq. (B.6) into Eq. (B.5) leaves

$$
\pi_{t}=\pi^{*}-\alpha \beta \gamma_{1}-\beta \gamma_{2} \alpha \eta x_{t-1}-\frac{\alpha\left(\lambda^{b}+\beta \gamma_{2}\right)}{1+\alpha^{2}\left(\lambda^{b}+\beta \gamma_{2}\right)} u_{t} .
$$

Substituting Eqs. (B.6) and (B.7) into Eq. (3) results in

$$
x_{t}=\eta x_{t-1}+\frac{1}{1+\alpha^{2}\left(\lambda^{b}+\beta \gamma_{2}\right)} u_{t}
$$


Now, computing $E_{t-1}\left[L_{t}^{b}+\beta V\left(x_{t}\right)\right]$ using the solutions in Eqs. (B.7) and (B.8) and comparing the coefficients of the result with $V\left(x_{t-1}\right)$ produces

$$
\begin{aligned}
& \left(1+\alpha^{2} \beta \gamma_{2}\right) \gamma_{1} \beta \eta=\gamma_{1} \text { and } \\
& \left(1+\alpha^{2} \beta \gamma_{2}\right) \gamma_{2} \beta \eta=\gamma_{2} \text {. Therefore, } \\
& \gamma_{1}=\gamma_{2}=0 \text { or } \gamma_{2}=\frac{1-\beta \eta}{\alpha^{2} \beta^{2} \eta} \text { and } \gamma_{1}=\text { anything. }
\end{aligned}
$$

Corresponding to $\gamma_{1}=\gamma_{2}=0$, the discretion solutions in Eqs. (B.7) and (B.8) become

$$
\begin{aligned}
& \pi_{t}=\pi^{*}-\frac{\alpha \lambda^{b}}{1+\alpha^{2} \lambda^{b}} u_{t}, \text { and } \\
& x_{t}=\eta x_{t-1}+\frac{1}{1+\alpha^{2} \lambda^{b}} u_{t} .
\end{aligned}
$$

\section{Appendix C: Discretionary Policy with Long-Run Target Values}

\section{in a Hybrid New-Keynesian Model}

The central bank operates with discretion, and always re-minimizes each period, subject to the Phillips curve (Eq. 13), the expectation of the intertemporal loss function

$$
\mathbb{L}^{b}=E_{0}\left(\sum_{t=0}^{\infty} \beta^{t} L_{t}^{b}\right)
$$

with the period loss function in Eq. (51).

Combining the Phillips curve and its equilibrium in Eqs. (13) and (24) defines the Phillips curve around its equilibrium

$$
\left(\pi_{t}-\bar{\pi}\right)=\kappa\left(x_{t}-\bar{x}\right)+\phi\left(\pi_{t-1}-\bar{\pi}\right)+(1-\phi) \beta\left(E_{t} \pi_{t+1}-\bar{\pi}\right)+u_{t}
$$

The Lagrangian expression of the problem equals 


$$
\mathfrak{L}=E_{0} \sum_{t=0}^{\infty} \beta^{t}\left\{\begin{array}{l}
\frac{1}{2}\left[\left(\pi_{t}-\bar{\pi}\right)^{2}+\lambda^{b}\left(x_{t}-\bar{x}\right)^{2}\right] \\
+\psi_{t}\left[\begin{array}{l}
\kappa\left(x_{t}-\bar{x}\right)+\phi\left(\pi_{t-1}-\bar{\pi}\right) \\
+(1-\phi) \beta\left(E_{t} \pi_{t+1}-\bar{\pi}\right)+u_{t}-\left(\pi_{t}-\bar{\pi}\right)
\end{array}\right]
\end{array}\right] .
$$

As the central bank re-formulates policy each period, we compute the first-order conditions with respect to $\pi_{0}, x_{0}$, and $x_{1}$, and eliminate the multipliers from the first-order conditions leads to the consolidated first-order condition

$$
\left(\pi_{0}-\bar{\pi}\right)=-\frac{\lambda^{b}}{\kappa}\left(x_{0}-\bar{x}\right)+\frac{\lambda^{b}}{\kappa} \phi \beta\left(E_{0} x_{1}-\bar{x}\right) .
$$

We write the consolidated first-order condition in $t$ period

$$
\left(\pi_{t}-\bar{\pi}\right)=-\frac{\lambda^{b}}{\kappa}\left(x_{t}-\bar{x}\right)+\frac{\lambda^{b}}{\kappa} \phi \beta\left(E_{t} x_{t+1}-\bar{x}\right) .
$$

Solve for $x_{t}$. Lagging and leading one period of Eq. (C.5) gives

$$
\begin{aligned}
& \left(\pi_{t-1}-\bar{\pi}\right)=-\frac{\lambda^{b}}{\kappa}\left(x_{t-1}-\bar{x}\right)+\frac{\lambda^{b}}{\kappa} \phi \beta\left(E_{t-1} x_{t}-\bar{x}\right), \text { and } \\
& \left(E_{t} \pi_{t+1}-\bar{\pi}\right)=-\frac{\lambda^{b}}{\kappa}\left(E_{t} x_{t+1}-\bar{x}\right)+\frac{\lambda^{b}}{\kappa} \phi \beta\left(E_{t} x_{t+2}-\bar{x}\right) .
\end{aligned}
$$

Substituting Eqs. (C.5) to (C.7) into the Phillips curve in Eq. (C.2), applying $E_{t-1}$ and arranging produces

$$
\begin{gathered}
a \beta^{2}\left(E_{t-1} x_{t+2}-\bar{x}\right)-\beta\left(E_{t-1} x_{t+1}-\bar{x}\right)+\left(1+\frac{\kappa^{2}}{\lambda^{b}}+\phi^{2} \beta\right)\left(E_{t-1} x_{t}-\bar{x}\right) \\
-\phi\left(x_{t-1}-\bar{x}\right)+\frac{\kappa}{\lambda^{b}} E_{t-1} u_{t}=0 .
\end{gathered}
$$

Assume that the solution of Eq. (C.8) takes the form

$$
\left(E_{t-1} x_{t}-\bar{x}\right)=\tilde{\delta}\left(x_{t-1}-\bar{x}\right)+f E_{t-1} u_{t} .
$$

Leading one and two periods of Eq. (C.9) and applying expectations as well as using 
$E_{t-1} u_{t+1}=\rho E_{t-1} u_{t}$ generate

$$
\left(E_{t-1} x_{t+1}-\bar{x}\right)=\tilde{\delta}\left(E_{t-1} x_{t}-\bar{x}\right)+f \rho E_{t-1} u_{t}, \text { and }
$$$$
\left(E_{t-1} x_{t+2}-\bar{x}\right)=\tilde{\delta}\left(E_{t-1} x_{t+1}-\bar{x}\right)+f \rho^{2} E_{t-1} u_{t}=\tilde{\delta}^{2}\left(E_{t-1} x_{t}-\bar{x}\right)+(\tilde{\delta}+\rho) f \rho E_{t-1} u_{t} .
$$

Substituting Eqs. (C.10) and (C.11) into (C.8) produces

$$
\left(E_{t-1} x_{t}-\bar{x}\right)=\frac{\phi\left(x_{t-1}-\bar{x}\right)+\left\{[1-a \beta(\tilde{\delta}+\rho)] f \beta \rho-\frac{\kappa}{\lambda^{b}}\right\} E_{t-1} u_{t}}{a \beta^{2} \tilde{\delta}^{2}-\beta \tilde{\delta}+\left(1+\frac{\kappa^{2}}{\lambda^{b}}+\phi^{2} \beta\right)} .
$$

Comparing Eq. (C.12) with Eq. (C.9) produces

$$
\begin{aligned}
& a \beta^{2} \tilde{\delta}^{3}-\beta \tilde{\delta}^{2}+\left(1+\frac{\kappa^{2}}{\lambda^{b}}+\phi^{2} \beta\right) \tilde{\delta}=\phi, \text { and } \\
& f=\frac{\tilde{\delta} \kappa}{\lambda^{b}\{[1-a \beta(\tilde{\delta}+\rho)] \tilde{\delta} \beta \rho-\phi\}} .
\end{aligned}
$$

Now, solve for $\pi_{t}$. By Eqs. (C.5) and (C.6), we can derive

$$
\left(E_{t-1} \pi_{t}-\bar{\pi}\right)-\tilde{\delta}\left(\pi_{t-1}-\bar{\pi}\right)=-\frac{\lambda^{b}}{\kappa}\left\{\begin{array}{l}
{\left[\left(E_{t-1} x_{t}-\bar{x}\right)-\tilde{\delta}\left(x_{t-1}-\bar{x}\right)\right]} \\
-\phi \beta\left[\left(E_{t-1} x_{t+1}-\bar{x}\right)-\tilde{\delta}\left(E_{t-1} x_{t}-\bar{x}\right)\right]
\end{array}\right\} .
$$

Substituting Eqs. (C.9) and (C.10) into Eq. (C.15) gives

$$
\left(E_{t-1} \pi_{t}-\bar{\pi}\right)=\tilde{\delta}\left(\pi_{t-1}-\bar{\pi}\right)-\frac{\lambda^{b}}{\kappa} f(1-\phi \beta \rho) E_{t-1} u_{t}
$$

where $\tilde{\delta}$ and $f$ are defined in Eqs. (C.13) and (C.14).

Eqs. (C.9) and (C.16) are the solutions. Does a less-than-one root exist for the characteristic equation (C.13)? Consider the two extreme cases of $\phi=0$ and $\phi=1$. For the case $\phi$ $=0$, the characteristic equation (C.13) reduces to $\beta \tilde{\delta}^{2}-\left(1+\kappa^{2} / \lambda^{b}\right) \tilde{\delta}=0$, and its two roots are 
$\tilde{\delta}=0$, which is a degenerative solution, and $\tilde{\delta}=\left(1+\kappa^{2} / \lambda^{b}\right) / \beta>1$. The system diverges when $\phi=0$.

For the case $\phi=1$, the characteristic equation (C.13) reduces to

$$
\beta \tilde{\delta}^{2}-\left(1+\frac{\kappa^{2}}{\lambda^{b}}+\beta\right) \tilde{\delta}+1=0
$$

We can show that the bigger root exceeds one and the smaller root lies between zero and one. The system can converge when $\phi=1$.

As a result, when the lag index $\phi$ goes from 0 to 1 , the system changes from divergence to convergence. Or, the system may diverge, converge, diverge, converge and so on. We can conclude that $\bar{\pi}$ and $\bar{x}$ are asymptotically controllable, if the characteristic equation (C.13) possesses a root that is less than one, $\tilde{\delta}<1$.

Furthermore, consider whether discretionary policy with the long-run target values can achieve path controllability (i.e., $\tilde{\delta}=\delta$ ). Now, derive the conditions for $\tilde{\delta}=\delta$, using the two characteristic equations, Eqs. (C.13) and (18a), under both discretionary policy and Ramsey policy. When $\tilde{\delta}=\delta$, the characteristic equation (Eq. C.13) becomes

$$
a \beta^{2} \delta^{3}-\beta \delta^{2}+\left(1+\frac{\kappa^{2}}{\lambda^{b}}+\phi^{2} \beta\right) \delta=\phi
$$

After substitution from Eq. (18c), the characteristic equation (18a) equals

$$
\left\{a \beta^{2} \delta^{3}-\beta \delta^{2}+\left[1+\frac{\kappa^{2}}{\lambda}+\phi^{2} \beta\right] \delta\right\} \delta+(1-\phi)^{2} \beta \delta^{2}-\delta+\phi(1-\phi)=0 .
$$

Assuming

$$
\lambda^{b}=\lambda
$$


and substituting Eq. (C.18) into Eq. (C.19) and arranging gives

$$
(1-\phi)\left[(1-\phi) \beta \delta^{2}-\delta+\phi\right]=0
$$

Eq. (C.21) gives rise to the conditions for $\tilde{\delta}=\delta$ and $\lambda^{b}=\lambda$

(C.22a) $\quad \phi=1$, and/or

$$
\phi\left(1-\beta \delta^{2}\right)=\delta-\beta \delta^{2}
$$

Now, prove that $\phi\left(1-\beta \delta^{2}\right) \neq \delta-\beta \delta^{2}$ for $\phi \in(0,1)$ by contradiction. Assume Eq.

(C.22b) holds. Substituting Eqs. (18b) and (18c) into Eq. (18a) and arranging produces

$$
\phi(1-\phi)\left(1-\beta \delta^{2}\right)^{2}+\delta\left(\delta-\beta \delta^{2}\right)-\left(\delta-\beta \delta^{2}\right)+\frac{\kappa^{2}}{\lambda} \delta^{2}=0
$$

Substituting Eq. (C.22b) into Eq. (C.23) and arranging produces

$$
\left(\delta-\beta \delta^{2}\right)\left[\delta-\beta \delta^{2}-\phi\left(1-\beta \delta^{2}\right)\right]+\frac{\kappa^{2}}{\lambda} \delta^{2}=0 .
$$

Using Eq. (C.22b), Eq. (C.24) reduces to $\kappa^{2} \delta^{2} / \lambda=0$. Thus, $\delta=0$. With $\delta=0$ the characteristic equation (18a) reduces to $a=0 . a=0$ contradicts with $\phi \in(0,1)$. Therefore, $\phi\left(1-\beta \delta^{2}\right) \neq \delta-\beta \delta^{2}$ for $\phi \in(0,1)$.

In sum, if $\lambda^{b}=\lambda$, then

$$
\tilde{\delta}=\delta \Leftrightarrow \phi=1
$$

We proved that $\tilde{\delta}=\delta \Rightarrow \phi=1$. We can easily show that $\phi=1 \Rightarrow \tilde{\delta}=\delta$ by noting that Eq. (C.13) equals Eq. (18a) when $\lambda^{b}=\lambda$ and $\phi=1$. Eq. (C.25) means that discretionary policy proves path controllable, when $\phi=1$. Now, we prove that the stabilization bias does not exist when $\phi=1$ under discretionary policy with $\left(\pi^{b}, x^{b}\right)=(\bar{\pi}, \bar{x})$ and $\lambda^{b}=\lambda$. 
With $\lambda^{b}=\lambda, \phi=1$, and $\tilde{\delta}=\delta$, Eq. (C.14) becomes

$$
f=-\frac{\kappa \delta}{\lambda(1-\delta \beta \rho)}
$$

From Eq. (C.16),

$$
\begin{aligned}
& \tilde{\pi}_{t}\left(u_{t}\right)=-\frac{\lambda}{\kappa} f(1-\beta \rho) u_{t}=\frac{\delta(1-\beta \rho)}{(1-\delta \beta \rho)} u_{t} . \text { Thus, } \\
& \left(\tilde{\pi}_{t}\left(u_{t}\right), \tilde{x}_{t}\left(u_{t}\right)\right)=\left(\frac{\delta(1-\beta \rho)}{(1-\delta \beta \rho)} u_{t},-\frac{\kappa \delta}{\lambda(1-\delta \beta \rho)} u_{t}\right) .
\end{aligned}
$$

With $\phi=1$, Eq. (22) reduces to

$$
\left(\bar{\pi}_{t}\left(u_{t}\right), \bar{x}_{t}\left(u_{t}\right)\right)=\left(\frac{1}{d}(1-\beta \rho) u_{t},-\frac{\kappa}{\lambda d} u_{t}\right),
$$

Noting $1 / d=\delta /(1-\delta \beta \rho)$ in Eq. (19) and comparing Eq. (C.28) with Eq. (C.29) yields

$$
\left(\tilde{\pi}_{t}\left(u_{t}\right), \tilde{x}_{t}\left(u_{t}\right)\right)=\left(\bar{\pi}_{t}\left(u_{t}\right), \bar{x}_{t}\left(u_{t}\right)\right)
$$

\section{Appendix D: Discretionary Policy with Lagged, Short-Run Target Values}

\section{in a Hybrid New-Keynesian Model}

The central bank operates with discretion, and always re-minimizes each period, subject to the Phillips curve (Eq. 13) or the Phillips curve around its equilibrium in Eq. (C.2), the expectation of the intertemporal loss function

$$
\mathbb{L}^{b}=E_{0}\left(\sum_{t=0}^{\infty} \beta^{t} L_{t}^{b}\right)
$$

with the period loss function in Eq. (62).

The Lagrangian expression of the problem equals 


$$
\mathfrak{L}=E_{0} \sum_{t=0}^{\infty} \beta^{t}\left\{\begin{array}{l}
\frac{1}{2}\left\{\begin{array}{l}
{\left[\left(\pi_{t}-\bar{\pi}\right)-\delta\left(\pi_{t-1}-\bar{\pi}\right)\right]^{2}} \\
+\lambda^{b}\left[\left(x_{t}-\bar{x}\right)-\delta\left(x_{t-1}-\bar{x}\right)\right]^{2}
\end{array}\right\} \\
+\psi_{t}\left[\begin{array}{l}
\kappa\left(x_{t}-\bar{x}\right)+\phi\left(\pi_{t-1}-\bar{\pi}\right) \\
+(1-\phi) \beta\left(E_{t} \pi_{t+1}-\bar{\pi}\right)+u_{t}-\left(\pi_{t}-\bar{\pi}\right)
\end{array}\right]
\end{array}\right\} .
$$

As the central bank re-formulates policy each period, we only calculate the first-order conditions with respect to $\pi_{0}, x_{0}$, and $x_{1}$, and eliminate the multipliers $\psi_{0}$ and $\psi_{1}$ leads to the consolidated first-order condition

$$
\begin{gathered}
\kappa\left\{\begin{array}{l}
{\left[\left(\pi_{0}-\bar{\pi}\right)-\delta\left(\pi_{-1}-\bar{\pi}\right)\right]} \\
-\beta \delta\left[\left(E_{0} \pi_{1}-\bar{\pi}\right)-\delta\left(\pi_{0}-\bar{\pi}\right)\right]
\end{array}\right\} \\
+\lambda^{b}\left\{\begin{array}{l}
{\left[\left(x_{0}-\bar{x}\right)-\delta\left(x_{-1}-\bar{x}\right)\right]} \\
-\beta(\phi+\delta)\left[\left(E_{0} x_{1}-\bar{x}\right)-\delta\left(x_{0}-\bar{x}\right)\right] \\
+\phi \beta^{2} \delta\left[\left(E_{0} x_{2}-\bar{x}\right)-\delta\left(E_{0} x_{1}-\bar{x}\right)\right]
\end{array}\right\}=0 .
\end{gathered}
$$

We write the consolidated first-order condition in $t$ period

$$
\begin{gathered}
\kappa\left\{\begin{array}{l}
{\left[\left(\pi_{t}-\bar{\pi}\right)-\delta\left(\pi_{t-1}-\bar{\pi}\right)\right]} \\
-\beta \delta\left[\left(E_{t} \pi_{t+1}-\bar{\pi}\right)-\delta\left(\pi_{t}-\bar{\pi}\right)\right]
\end{array}\right\} \\
+\lambda^{b}\left\{\begin{array}{l}
{\left[\left(x_{t}-\bar{x}\right)-\delta\left(x_{t-1}-\bar{x}\right)\right]} \\
-\beta(\phi+\delta)\left[\left(E_{t} x_{t+1}-\bar{x}\right)-\delta\left(x_{t}-\bar{x}\right)\right] \\
+\phi \beta^{2} \delta\left[\left(E_{t} x_{t+2}-\bar{x}\right)-\delta\left(E_{t} x_{t+1}-\bar{x}\right)\right]
\end{array}\right\}=0
\end{gathered}
$$

Transforming the Phillips curve in Eq. (C.2) and lagging one and leading one and two period produces

$$
\begin{aligned}
& \kappa\left(x_{t}-\bar{x}\right)=\left(\pi_{t}-\bar{\pi}\right)-\phi\left(\pi_{t-1}-\bar{\pi}\right)-(1-\phi) \beta\left(E_{t} \pi_{t+1}-\bar{\pi}\right)-u_{t}, \\
& \kappa\left(x_{t-1}-\bar{x}\right)=\left(\pi_{t-1}-\bar{\pi}\right)-\phi\left(\pi_{t-2}-\bar{\pi}\right)-(1-\phi) \beta\left(E_{t-1} \pi_{t}-\bar{\pi}\right)-u_{t-1}, \\
& \kappa\left(E_{t} x_{t+1}-\bar{x}\right)=\left(E_{t} \pi_{t+1}-\bar{\pi}\right)-\phi\left(\pi_{t}-\bar{\pi}\right)-(1-\phi) \beta\left(E_{t} \pi_{t+2}-\bar{\pi}\right)-E_{t} u_{t+1}, \text { and }
\end{aligned}
$$


(D.5d)

$$
\kappa\left(E_{t} x_{t+2}-\bar{x}\right)=\left(E_{t} \pi_{t+2}-\bar{\pi}\right)-\phi\left(E_{t} \pi_{t+1}-\bar{\pi}\right)-(1-\phi) \beta\left(E_{t} \pi_{t+3}-\bar{\pi}\right)-E_{t} u_{t+2} .
$$

Using Eqs. (D.5a) to (D.5d), compute

(D.6a)

$$
\begin{aligned}
& \kappa\left[\left(x_{t}-\bar{x}\right)-\delta\left(x_{t-1}-\bar{x}\right)\right]= \\
& {\left[\left(\pi_{t}-\bar{\pi}\right)-\delta\left(\pi_{t-1}-\bar{\pi}\right)\right]-\phi\left[\left(\pi_{t-1}-\bar{\pi}\right)-\delta\left(\pi_{t-2}-\bar{\pi}\right)\right],}
\end{aligned}
$$$$
-(1-\phi) \beta\left[\left(E_{t} \pi_{t+1}-\bar{\pi}\right)-\delta\left(E_{t-1} \pi_{t}-\bar{\pi}\right)\right]-\left(u_{t}-\delta u_{t-1}\right) \text {, }
$$

(D.6b)

$$
\begin{aligned}
& \kappa\left[\left(E_{t} x_{t+1}-\bar{x}\right)-\delta\left(x_{t}-\bar{x}\right)\right]= \\
& {\left[\left(E_{t} \pi_{t+1}-\bar{\pi}\right)-\delta\left(\pi_{t}-\bar{\pi}\right)\right]-\phi\left[\left(\pi_{t}-\bar{\pi}\right)-\delta\left(\pi_{t-1}-\bar{\pi}\right)\right]} \\
& -(1-\phi) \beta\left[\left(E_{t} \pi_{t+2}-\bar{\pi}\right)-\delta\left(E_{t} \pi_{t+1}-\bar{\pi}\right)\right]-\left(E_{t} u_{t+1}-\delta u_{t}\right), \text { and }
\end{aligned}
$$

$$
\kappa\left[\left(E_{t} x_{t+2}-\bar{x}\right)-\delta\left(E_{t} x_{t+1}-\bar{x}\right)\right]=
$$

(D.6c)

$$
\begin{aligned}
& {\left[\left(E_{t} \pi_{t+2}-\bar{\pi}\right)-\delta\left(E_{t} \pi_{t+1}-\bar{\pi}\right)\right]-\phi\left[\left(E_{t} \pi_{t+1}-\bar{\pi}\right)-\delta\left(\pi_{t}-\bar{\pi}\right)\right]} \\
& -(1-\phi) \beta\left[\left(E_{t} \pi_{t+3}-\bar{\pi}\right)-\delta\left(E_{t} \pi_{t+2}-\bar{\pi}\right)\right]-\left(E_{t} u_{t+2}-\delta E_{t} u_{t+1}\right) .
\end{aligned}
$$

Multiplying Eq. (D.4) by $\kappa$ and substituting Eqs. (D.6a) to (D.6c) into it produces

$$
\begin{aligned}
& \kappa^{2}\left\{\begin{array}{l}
{\left[\left(\pi_{t}-\bar{\pi}\right)-\delta\left(\pi_{t-1}-\bar{\pi}\right)\right]} \\
-\beta \delta\left[\left(E_{t} \pi_{t+1}-\bar{\pi}\right)-\delta\left(\pi_{t}-\bar{\pi}\right)\right]
\end{array}\right\}
\end{aligned}
$$

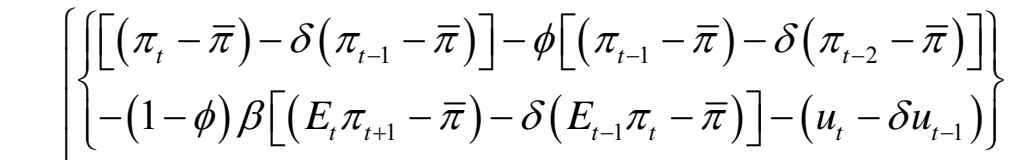

$$
\begin{aligned}
& +\lambda^{b}\left\{\begin{array}{r}
-\beta(\phi+\delta)\left\{\begin{array}{l}
{\left[\left(E_{t} \pi_{t+1}-\bar{\pi}\right)-\delta\left(\pi_{t}-\bar{\pi}\right)\right]-\phi\left[\left(\pi_{t}-\bar{\pi}\right)-\delta\left(\pi_{t-1}-\bar{\pi}\right)\right]} \\
-(1-\phi) \beta\left[\left(E_{t} \pi_{t+2}-\bar{\pi}\right)-\delta\left(E_{t} \pi_{t+1}-\bar{\pi}\right)\right]-\left(E_{t} u_{t+1}-\delta u_{t}\right)
\end{array}\right\} \\
+\phi \beta^{2} \delta\left\{\begin{array}{l}
{\left[\left(E_{t} \pi_{t+2}-\bar{\pi}\right)-\delta\left(E_{t} \pi_{t+1}-\bar{\pi}\right)\right]-\phi\left[\left(E_{t} \pi_{t+1}-\bar{\pi}\right)-\delta\left(\pi_{t}-\bar{\pi}\right)\right]} \\
-(1-\phi) \beta\left[\left(E_{t} \pi_{t+3}-\bar{\pi}\right)-\delta\left(E_{t} \pi_{t+2}-\bar{\pi}\right)\right]-\left(E_{t} u_{t+2}-\delta E_{t} u_{t+1}\right)
\end{array}\right\}
\end{array}\right\}=0 .
\end{aligned}
$$

Assume that $u_{t}=0$ and the solution without shocks, $u_{t}$, takes the form

$$
\left(\pi_{t}-\bar{\pi}\right)=\tilde{\delta}\left(\pi_{t-1}-\bar{\pi}\right)
$$

Using Eq. (D.8) and $u_{t}=0$, Eq. (D.7) becomes 
(D.9)

$$
\begin{aligned}
& \kappa^{2}\left[(\tilde{\delta}-\delta)\left(\pi_{t-1}-\bar{\pi}\right)-\beta \delta(\tilde{\delta}-\delta)\left(\pi_{t}-\bar{\pi}\right)\right] \\
& +\lambda^{b}\left\{\begin{array}{l}
{\left[\begin{array}{l}
(\tilde{\delta}-\delta)\left(\pi_{t-1}-\bar{\pi}\right)-\phi(\tilde{\delta}-\delta)\left(\pi_{t-2}-\bar{\pi}\right) \\
-(1-\phi) \beta(\tilde{\delta}-\delta)\left(\pi_{t}-\bar{\pi}\right)
\end{array}\right]} \\
-\beta(\phi+\delta)\left[\begin{array}{l}
(\tilde{\delta}-\delta)\left(\pi_{t}-\bar{\pi}\right)-\phi(\tilde{\delta}-\delta)\left(\pi_{t-1}-\bar{\pi}\right) \\
-(1-\phi) \beta(\tilde{\delta}-\delta)\left(\pi_{t+1}-\bar{\pi}\right)
\end{array}\right] \\
+\phi \beta^{2} \delta\left[\begin{array}{l}
(\tilde{\delta}-\delta)\left(\pi_{t+1}-\bar{\pi}\right)-\phi(\tilde{\delta}-\delta)\left(\pi_{t}-\bar{\pi}\right) \\
-(1-\phi) \beta(\tilde{\delta}-\delta)\left(\pi_{t+2}-\bar{\pi}\right)
\end{array}\right]
\end{array}\right\}=0 .
\end{aligned}
$$

Obviously, $\tilde{\delta}=\delta$ solves Eq. (D.9). Now, Eq. (D.8) equals

$$
\left(\pi_{t}-\bar{\pi}\right)=\delta\left(\pi_{t-1}-\bar{\pi}\right) .
$$

Using Eq. (D.10), (D.4) reduces to

$$
\left\{\begin{array}{c}
{\left[\left(x_{t}-\bar{x}\right)-\delta\left(x_{t-1}-\bar{x}\right)\right]-\beta(\phi+\delta)\left[\left(E_{t} x_{t+1}-\bar{x}\right)-\delta\left(x_{t}-\bar{x}\right)\right]} \\
+\phi \beta^{2} \delta\left[\left(E_{t} x_{t+2}-\bar{x}\right)-\delta\left(E_{t} x_{t+1}-\bar{x}\right)\right]
\end{array}\right\}=0 .
$$

Obviously, one of the solutions of Eq. (D.11) equals

$$
\left(x_{t}-\bar{x}\right)=\delta\left(x_{t-1}-\bar{x}\right) .
$$

By Eqs. (D.10) and (D.12), the discretionary policy proves path controllable.

When $u_{t} \neq 0$, the stabilization bias exists. Now, determine the weight parameter, $\lambda^{b}$, to see if a proper weight can eliminate the stabilization bias. To eliminate biases, $\tilde{\pi}_{t}\left(u_{t}\right)=\bar{\pi}_{t}\left(u_{t}\right)$ and $\tilde{x}_{t}\left(u_{t}\right)=\bar{x}_{t}\left(u_{t}\right)$ must hold. That is, Eqs. (17a) and (17b) must satisfy the consolidated firstorder condition (Eq. D.4). Applying $E_{t-1}$ to Eq. (D.4) and using Eqs. (17a) and (17b) determine

$$
\lambda^{b}=\left(\phi-\phi_{c}\right) \frac{\left(1-\beta \rho^{2}\right)}{\rho(1-\phi \beta \rho)} \lambda .
$$




\section{Appendix E: Discretionary Policy with Expected, Short-Run Inflation Target Value in a Hybrid New-Keynesian Model}

The expectation of the intertemporal loss function equals

$$
\mathbb{L}^{b}=E_{0}\left(\sum_{t=0}^{\infty} \beta^{t} L_{t}^{b}\right),
$$

with the period loss function in Eq. (67).

The Lagrangian expression of the problem equals

$$
\mathfrak{L}=E_{0} \sum_{t=0}^{\infty} \beta^{t}\left\{\begin{array}{l}
\frac{1}{2}\left\{\begin{array}{l}
{\left[\left(\pi_{t}-\bar{\pi}\right)-\delta^{-1}\left(\pi_{t+1}-\bar{\pi}\right)\right]^{2}} \\
+\lambda^{b}\left[\left(x_{t}-\bar{x}\right)-\delta\left(x_{t-1}-\bar{x}\right)\right]^{2}
\end{array}\right\} \\
+\psi_{t}\left[\begin{array}{l}
\kappa\left(x_{t}-\bar{x}\right)+\phi\left(\pi_{t-1}-\bar{\pi}\right) \\
+(1-\phi) \beta\left(E_{t} \pi_{t+1}-\bar{\pi}\right)+u_{t}-\left(\pi_{t}-\bar{\pi}\right)
\end{array}\right]
\end{array}\right\} .
$$

As the central bank re-makes policy each period, we only calculate the first-order conditions with respect to $\pi_{0}, x_{0}$, and $x_{1}$, and eliminate the multipliers $\psi_{0}$ and $\psi_{1}$ leads to the consolidated first-order condition

$$
\kappa\left[\left(\pi_{0}-\bar{\pi}\right)-\delta^{-1}\left(E_{0} \pi_{1}-\bar{\pi}\right)\right]+\lambda^{b}\left\{\begin{array}{l}
{\left[\left(x_{0}-\bar{x}\right)-\delta\left(x_{-1}-\bar{x}\right)\right]} \\
-\beta(\delta+\phi)\left[\left(E_{0} x_{1}-\bar{x}\right)-\delta\left(x_{0}-\bar{x}\right)\right] \\
+\phi \beta^{2} \delta\left[\left(E_{0} x_{2}-\bar{x}\right)-\delta\left(x_{1}-\bar{x}\right)\right]
\end{array}\right\}=0 .
$$

We write the consolidated first-order condition in $t$ period. Multiplying the condition by $\delta$ and transforming gives

$$
\kappa\left[\left(E_{t} \pi_{t+1}-\bar{\pi}\right)-\delta\left(\pi_{t}-\bar{\pi}\right)\right]=\lambda^{b} \delta\left\{\begin{array}{l}
{\left[\left(x_{t}-\bar{x}\right)-\delta\left(x_{t-1}-\bar{x}\right)\right]} \\
-\beta(\delta+\phi)\left[\left(E_{t} x_{t+1}-\bar{x}\right)-\delta\left(x_{t}-\bar{x}\right)\right] \\
+\phi \beta^{2} \delta\left[\left(E_{t} x_{t+2}-\bar{x}\right)-\delta\left(x_{t+1}-\bar{x}\right)\right]
\end{array}\right\} .
$$

Multiplying Eq. (E.4) by $\kappa$ and substituting Eqs. (D.6a) to (D.6c) into it produces 
(E.5)

$$
\left.\begin{array}{rl}
\kappa^{2}\left[\left(E_{t} \pi_{t+1}-\bar{\pi}\right)-\delta\left(\pi_{t}-\bar{\pi}\right)\right]= \\
\lambda^{b} \delta\left\{\begin{array}{l}
\left\{\left(\pi_{t}-\bar{\pi}\right)-\delta\left(\pi_{t-1}-\bar{\pi}\right)\right]-\phi\left[\left(\pi_{t-1}-\bar{\pi}\right)-\delta\left(\pi_{t-2}-\bar{\pi}\right)\right] \\
-(1-\phi) \beta\left[\left(E_{t} \pi_{t+1}-\bar{\pi}\right)-\delta\left(E_{t-1} \pi_{t}-\bar{\pi}\right)\right]-\left(u_{t}-\delta u_{t-1}\right)
\end{array}\right\} \\
-\beta(\delta+\phi)\left\{\begin{array}{l}
{\left[\left(E_{t} \pi_{t+1}-\bar{\pi}\right)-\delta\left(\pi_{t}-\bar{\pi}\right)\right]-\phi\left[\left(\pi_{t}-\bar{\pi}\right)-\delta\left(\pi_{t-1}-\bar{\pi}\right)\right]} \\
-(1-\phi) \beta\left[\left(E_{t} \pi_{t+2}-\bar{\pi}\right)-\delta\left(E_{t} \pi_{t+1}-\bar{\pi}\right)\right]-\left(E_{t} u_{t+1}-\delta u_{t}\right)
\end{array}\right\} \\
+\phi \beta^{2} \delta\left\{\begin{array}{l}
{\left[\left(E_{t} \pi_{t+2}-\bar{\pi}\right)-\delta\left(E_{t} \pi_{t+1}-\bar{\pi}\right)\right]-\phi\left[\left(E_{t} \pi_{t+1}-\bar{\pi}\right)-\delta\left(\pi_{t}-\bar{\pi}\right)\right]} \\
-(1-\phi) \beta\left[\left(E_{t} \pi_{t+3}-\bar{\pi}\right)-\delta\left(E_{t} \pi_{t+2}-\bar{\pi}\right)\right]-\left(E_{t} u_{t+2}-\delta E_{t} u_{t+1}\right)
\end{array}\right\}
\end{array}\right\} .
$$

Assume $u_{t}=0$ and the solution takes the form

$$
\left(\pi_{t}-\bar{\pi}\right)=\tilde{\delta}\left(\pi_{t-1}-\bar{\pi}\right) .
$$

When $u_{t}=0$, Eq. (E.5) becomes

(E.7)

$$
\left.\begin{array}{c}
\kappa^{2}\left[(\tilde{\delta}-\delta)\left(\pi_{t}-\bar{\pi}\right)\right]= \\
\lambda^{b} \delta\left\{\left\{\left[\begin{array}{l}
\left.[\tilde{\delta}-\delta)\left(\pi_{t-1}-\bar{\pi}\right)\right]-\phi\left[(\tilde{\delta}-\delta)\left(\pi_{t-2}-\bar{\pi}\right)\right] \\
-(1-\phi) \beta\left[(\tilde{\delta}-\delta)\left(\pi_{t}-\bar{\pi}\right)\right]
\end{array}\right\}\right.\right. \\
-\beta(\delta+\phi)\left\{\begin{array}{l}
{\left[(\tilde{\delta}-\delta)\left(\pi_{t}-\bar{\pi}\right)\right]-\phi\left[(\tilde{\delta}-\delta)\left(\pi_{t-1}-\bar{\pi}\right)\right]} \\
-(1-\phi) \beta\left[(\tilde{\delta}-\delta)\left(\pi_{t+1}-\bar{\pi}\right)\right]
\end{array}\right\} \\
+\phi \beta^{2} \delta\left\{\begin{array}{l}
{\left[(\tilde{\delta}-\delta)\left(\pi_{t+1}-\bar{\pi}\right)\right]-\phi\left[(\tilde{\delta}-\delta)\left(\pi_{t}-\bar{\pi}\right)\right]} \\
-(1-\phi) \beta\left[(\tilde{\delta}-\delta)\left(\pi_{t+2}-\bar{\pi}\right)\right]
\end{array}\right\}
\end{array}\right\} .
$$

Obviously, $\tilde{\delta}=\delta$ solves Eq. (E.7). Now, Eq. (E.6) equals

$$
\left(\pi_{t}-\bar{\pi}\right)=\delta\left(\pi_{t-1}-\bar{\pi}\right) .
$$

Using Eq. (E.8), (E.4) reduces to

$$
\left\{\left[\begin{array}{c}
{\left[\left(x_{t}-\bar{x}\right)-\delta\left(x_{t-1}-\bar{x}\right)\right]-\beta(\delta+\phi)\left[\left(E_{t} x_{t+1}-\bar{x}\right)-\delta\left(x_{t}-\bar{x}\right)\right]} \\
+\phi \beta^{2} \delta\left[\left(E_{t} x_{t+2}-\bar{x}\right)-\delta\left(x_{t+1}-\bar{x}\right)\right]
\end{array}\right\}=0 .\right.
$$

Obviously, one of the solutions of Eq. (E.9) equals 


$$
\left(x_{t}-\bar{x}\right)=\delta\left(x_{t-1}-\bar{x}\right)
$$

By Eqs. (E.8) and (E.10), the discretionary policy proves path controllable.

When $u_{t} \neq 0$, the stabilization bias arises. Now, determine the weight parameter, $\lambda^{b}$, to see if a proper weight can eliminate the stabilization bias. To eliminate biases, $\tilde{\pi}_{t}\left(u_{t}\right)=\bar{\pi}_{t}\left(u_{t}\right)$ and $\tilde{x}_{t}\left(u_{t}\right)=\bar{x}_{t}\left(u_{t}\right)$ must hold. That is, Eqs. (17a) and (17b) must satisfy the consolidated firstorder condition (Eq. E.4). Applying $E_{t-1}$ to Eq. (E.4) and using Eqs. (17a) and (17b) determine

$$
\lambda^{b}=\frac{\left(\phi_{c}-\phi\right)\left(1-\beta \rho^{2}\right)}{\delta(1-\delta \beta \rho)(1-\phi \beta \rho)} \lambda .
$$

\section{Appendix F: The Delegation in the Hybrid New-Keynesian Model with $\beta=0$}

When the discount factor equals zero $(\beta=0)$, the social loss function equals

$$
L_{t}=\frac{1}{2}\left[\left(\pi_{t}-\pi^{*}\right)^{2}+\lambda\left(x_{t}-x^{*}\right)^{2}\right]
$$

and the hybrid new-Keynesian Phillips curve equals

$$
\pi_{t}=\kappa X_{t}+\phi \pi_{t-1}+u_{t}
$$

If $\phi=0$, the model is apparently static. If $\phi \neq 0$, the model is essentially static, since the central bank only cares about the present period loss and we can treat $\pi_{t-1}$ as a predetermined variable. In addition, the model in Eqs. (F.1) and (F.2) contains no expectations and, thus, the timeinconsistency problem does not exist.

The Lagrangian expression of the problem equals

$$
\mathfrak{L}=\frac{1}{2}\left[\left(\pi_{t}-\pi^{*}\right)^{2}+\lambda\left(x_{t}-x^{*}\right)^{2}\right]+\psi_{t}\left(\kappa x_{t}+\phi \pi_{t-1}+u_{t}-\pi_{t}\right) .
$$

Computing the first-order conditions with respect to $\pi_{t}$ and $x_{t}$ and eliminating the multiplier $\psi_{t}$ leads to the consolidated first-order condition 


$$
\lambda\left(x_{t}-x^{*}\right)+\kappa\left(\pi_{t}-\pi^{*}\right)=0 .
$$

Simultaneously solving the consolidated first-order condition (Eq. F.4) and the Phillips curve (Eq. F.2) produces

$$
\begin{aligned}
& \pi_{t}=\bar{\pi}+\frac{\lambda}{\lambda+\kappa^{2}} u_{t}, \text { and } \\
& x_{t}=\bar{x}-\frac{\kappa}{\lambda+\kappa^{2}} u_{t}, \text { where } \\
& \bar{\pi} \equiv \frac{\kappa\left(\lambda x^{*}+\kappa \pi^{*}\right)}{\lambda+\kappa^{2}}+\frac{\lambda \phi \pi_{t-1}}{\lambda+\kappa^{2}}, \text { and } \\
& \bar{x} \equiv \frac{\lambda x^{*}+\kappa \pi^{*}}{\lambda+\kappa^{2}}-\frac{\kappa \phi \pi_{t-1}}{\lambda+\kappa^{2}} .
\end{aligned}
$$

$\bar{\pi}$ and $\bar{x}$ are the equilibriums under the social loss function (F.1). Generally, $\pi^{*} \neq \bar{\pi}$ and $x^{*} \neq \bar{X}$. That is, $\pi^{*}$ and $x^{*}$ are not controllable.

Now, assume the central bank loss function as follows

$$
L_{t}^{b}=\frac{1}{2}\left[\left(\pi_{t}-\pi^{b}\right)^{2}+\lambda^{b}\left(x_{t}-x^{b}\right)^{2}\right] .
$$

Under the loss function (Eq. F.7), the optimal outcomes, which mimic Eqs. (F.5a) and (F.5b), equal

$$
\begin{aligned}
& \pi_{t}=\frac{\kappa\left(\lambda^{b} x^{b}+\kappa \pi^{b}\right)}{\lambda^{b}+\kappa^{2}}+\frac{\lambda^{b} \phi \pi_{t-1}}{\lambda^{b}+\kappa^{2}}+\frac{\lambda^{b}}{\lambda^{b}+\kappa^{2}} u_{t}, \text { and } \\
& x_{t}=\frac{\lambda^{b} x^{b}+\kappa \pi^{b}}{\lambda^{b}+\kappa^{2}}-\frac{\kappa \phi \pi_{t-1}}{\lambda^{b}+\kappa^{2}}-\frac{\kappa}{\lambda^{b}+\kappa^{2}} u_{t} .
\end{aligned}
$$

To eliminate the stabilization bias, comparing Eq. (F.8a) with Eq. (F.5a) produces

$$
\lambda^{b}=\lambda
$$

Adopt

$$
\pi^{b}=\bar{\pi} \text { and } x^{b}=\bar{x} .
$$


Using Eqs. (F.9), (F.10), (F.6a) and (F.6b), Eqs. (F.8a) and (F.8b) equal Eqs. (5a) and (5b).

That is, $\bar{\pi}$ and $\bar{x}$ are jointly controllable and stabilization bias disappears under the loss function in Eq. (F.7) with $\pi^{b}=\bar{\pi}, x^{b}=\bar{x}$, and $\lambda^{b}=\lambda$.

\section{References}

Acocella N. and Di Bartolomeo, G., (2006). Tinbergen and Theil Meet Nash: Controllability in Policy Games. Economics Letters 90(2), 213-218.

Acocella N., Di Bartolomeo, G., and Hughes Hallett, A., (2006). Controllability in Policy Games: Policy Neutrality and the Theory of Economic Policy Revisited. Computational Economics, 28(2), 91-112.

Acocella N., Di Bartolomeo, G., and Hughes Hallett, A., (2007). Controllability with Overlapping Targets: Or Why Target Independence May Not Be Good for You. Macroeconomic Dynamics 11(2), 202-213.

Aoki, M., (1975). On a Generalization of Tinbergen's Condition in the Theory of Policy to Dynamic Models. Review of Economic Studies 42(2), 293-296.

Arrow, K. J., (1951). Social Choice and Individual Values. Cowles Foundation Monograph 12, New York: J. Wiley.

Beetsma, R., and Jensen, H., (1999). Optimal Inflation Targets, 'Conservative' Central Banks, and Linear Inflation Contracts: Comment. American Economic Review 89(1), 342-347.

Brockett, R. W., and Mesarović, M. D., (1965). The Reproducibility of Multivariable Systems. Journal of Mathematical Analysis and Applications 11, 548-563.

Buiter, W. H., and Gersovitz, M., (1981). Issues in Controllability and the Theory of Economic Policy, Journal of Public Economics 15(1), 33-43.

Buiter, W. H., and Gersovitz, M., (1984). Controllability and the Theory of Economic Policy: A Further Note. Journal of Public Economics 24(1), 127-129.

Calvo, G. A., (1978). On the Time Consistency of Optimal Policy in a Monetary Economy. Econometrica 46(6), 1411-1428.

Chortareas, G. E., Aand Miller, S. M., (2003). Monetary Policy Delegation, Contract Costs, and Contract Targets. Bulletin of Economic Research, 55(1), pp. 101-112.

Clarida, R.; Gali, J., and Gertler, M., (1999). The Science of Monetary Policy: A New Keynesian Perspective. Journal of Economic Literature 37(4), 1661-1707. 
Hughes Hallett, A., Acocella, N., and Di Bartolomeo, G., (2010). Policy Games, Policy Neutrality and Tinbergen Controllability under Rational Expectations. Journal of Macroeconomics 32(1), 55-67.

Jensen, H., (2002). Targeting Nominal Income Growth or Inflation? American Economic Review 92(4), 928-956.

Kalman, R. E., (1960). Contributions to the Theory of Optimal Control Boletin de la Sociedad Matematica Mexicana 5, 102-119.

Kydland, F. E., and Prescott, E. C., (1977). Rules Rather Than Discretion: The Inconsistency of Optimal Plans. Journal of Political Economy 85(3), 473-491.

Maas, W. C. A., and Nijmeijer, H., (1994). Dynamic Path controllability in Economic Models. Journal of Economic Dynamics and Control 18(3-4), 781-805.

Nyberg, L., and Viotti, S., (1978). Controllability and the Theory of Economic Policy. A Critical View. Journal of Public Economics 9(1), 73-81.

Phillips, A. W., (1954). Stabilisation Policy in a Closed Economy. The Economic Journal 64(254), 290-323.

Preston, A. J., (1974). A Dynamic Generalization of Tinbergen's Theory of Policy. Review of Economic Studies 41(1), 65-74.

Rogoff, K., (1985). The Optimal Degree of Commitment to an Intermediate Monetary Target. Quarterly Journal of Economics 100(4), 1169-1189.

Svensson, L. E. O., (1997). Optimal Inflation Targets, 'Conservative' Central Banks, and Linear Inflation Contracts. American Economic Review 87(1), 98-114.

Svensson, L.E.O., and M. Woodford., (2005). Implementing Optimal Policy through InflationForecast Targeting. In The Inflation-Targeting Debate, ed. B. S. Bernanke and M. Woodford, 19-83. Chicago: University of Chicago Press.

Rawls, J., (1971). A Theory of Justice, Cambridge, MA: Harvard University Press.

Tinbergen, J., (1952). On the Theory of Economic Policy. Amsterdam: North Holland.

Tinbergen, J., (1963). On the theory of economic policy. (North-Holland, Amsterdam).

Tondini, G., (1984). Further Discussion on Controllability and the Theory of Economic Policy. Journal of Public Economics 24(1), 123-125.

Vestin, D., (2006). Price-Level versus Inflation Targeting. Journal of Monetary Economics 53(7), $1-16$.

Walsh, C. E., (1995). Optimal Contracts for Central Bankers. American Economic Review 85(1), 150-167. 
Wohltmann, H.-W., (1981). Complete, Perfect, and Maximal Controllability of Discrete Economic Systems. Zeitschrift fiir Nationalkonomie 41(1-2), 39-58.

Wohltmann, H.-W., (1985). On the Controllability of Continuous-Time Macroeconomic Models. Zeitschrift fiir Nationalkonomie 45(1), 47-66.

Woodford, M., (1999). Commentary: How Should Monetary Policy Be Conducted in an Era of Price Stability? In New Challenges for Monetary Policy, Kansas City: Federal Reserve Bank of Kansas City, 277-316.

Yuan, H., and Miller, S. M., (2010). Implementing Optimal Monetary Policy: Objectives and Rules. Economic Modelling 27(3), 737-745.

Yuan, H., Miller, S. M., and Chen, L. (2011). The Optimality and Controllability of Monetary Policy through Delegation with Consistent Targets. Scottish Journal of Political Economy 58(1), 82-106. 